\title{
GENERALIZED SECOND ORDER DIFFERENTIAL OPERATORS AND THEIR LATERAL CONDITIONS
}

\author{
By William Feller ${ }^{1}$
}

\section{Introduction}

The differential operator

$$
\mathfrak{A}=a D_{s}^{2}+b D_{s}, \quad a>0, \quad D_{s}=\frac{d}{d s},
$$

defined on an interval $s_{1}<s<s_{2}$ may be reduced to the form

$$
\mathfrak{A}=\alpha D_{x}^{2},
$$

by introducing a new variable $x$, namely, a solution of $\mathfrak{A} x=0$. All solutions of this differential equation are monotonic, and $x$ is determined up to a linear transformation. Putting

$$
m(x)=\int_{x_{0}}^{x} \alpha^{-1}(t) d t
$$

we can further reduce $\mathfrak{A}$ to a succession of two differentiations

$$
\mathfrak{A}=D_{m} D_{x} \text {. }
$$

It turns out that the canonical form (1.4) has considerable formal advantages over (1.1).

It will be observed that (1.4) is much more general than (1.1). In fact, the operator $\mathfrak{A}=D_{m} D_{x}$ is meaningful for an arbitrary strictly increasing function $m$, not necessarily continuous or bounded. (See Section 2 for the definitions.) This generalization introduces a remarkable simplification and unification of the theory. ${ }^{2}$ It is usual to transform (1.1) in several arbitrary ways, but the

Received March 28, 1957.

${ }_{1}^{1}$ This research was done while the author was on sabbatical leave of absence with the support of the United States Air Force through the Air Force Office of Scientific Research of the Air Research and Development Command.

${ }^{2}$ One has frequently to deal with functions defined, in two contiguous intervals, by two differential equations joined by so-called "transition conditions". In the new notation such a "system" reduces to one differential equation, and no transition conditions occur. For a simple example define $x$ and $m$ by

$$
x(s)=\left\{\begin{array}{c}
s \text { for } s<0 \\
2 s \text { for } s>0, \quad m(s)=s .
\end{array}\right.
$$

Then (1.4) reduces to $D_{s}^{2}$ when $s<0$ and $\frac{1}{2} D_{s}^{2}$ when $s>0$, with the "transition condition" $D_{s}^{-} \dot{f}(0)=\frac{1}{2} D_{s}^{+} f(0)$. Interchanging $x$ and $m$ in $\left(^{*}\right)$ leads to the same differential system with the transition condition $D_{s}^{-} f(0)=D_{s}^{+} f(0)$. A yearly crop of papers devoted to series expansions under conditions of this kind now becomes redundant, since the same general eigenfunction expansion is valid for all our operators. Moreover, due to the intrinsic characterization of the operators (1.4), it is possible to write down all possible transition conditions and render them more plausible. 
scale $x$ and the "canonical measure" $m$ are essentially unique and intrinsically related to the operator (1.4). The latter plays an important role in the theory of the vibrating string, $m$ representing the distribution of the actual mass. With the new notation, strings with a positive mass concentrated at individual points require no special treatment. As a matter of curiosity it is possible to treat the motion of a continuous string with the entire mass concentrated at the rational points. ${ }^{3}$

It is easy to understand why a treatment of differential operators in the special form (1.1) is never satisfactory. The formal adjoint of (1.1) is given by $\mathfrak{I}^{*} g=D_{s}^{2}(a g)-D_{s}(b g)$ provided the coefficients $a$ and $b$ are sufficiently regular. Without this highly undesirable proviso no formal adjoint of the form (1.1) exists. By contrast, no difficulties arise in connection with (1.4). Considered as operator on functions continuous in an interval $\beta_{1} \leqq x \leqq \beta_{2}$, it admits of the formal adjoint $\mathfrak{2}^{*}=D_{x} D_{m}$, which is an operator on measures. (The true adjoint will be discussed in Section 13.)

A perhaps more familiar point of view consists in considering $\mathfrak{A}$ as an operator in the traditional Hilbert space $\mathbf{H}_{m}$ defined by the norm

$$
\|f\|_{m}^{2}=\int_{\beta_{1+}}^{\beta_{2^{-}-}} f^{2}(x) d m(x) .
$$

The operator $\mathfrak{A}$ then becomes formally self-adjoint. In Section 10 we shall discuss how to make $\mathfrak{A}$ a true self-adjoint operator. In Section 11 we shall take up the same problem for the more desirable (though less familiar) Hilbert space $\overline{\mathbf{H}}_{m}$ over the closed interval $\beta_{1} \leqq x \leqq \beta_{2}$ when $m$ is extended to the boundaries by attributing a positive weight $m_{i}$ to $\beta_{i}$; the norm in this space is defined by

$$
\overline{\|f\|_{m}^{2}}=\|f\|_{m}^{2}+m_{1} f^{2}\left(\beta_{1}\right)+m_{2} f^{2}\left(\beta_{2}\right) .
$$

The operators of the form (1.4) are still of a special form, and one is automatically led to the study of the more general operator defined by $9 f f+c f$, where $c$ is a function. However, the class of operators of this type does not admit of an elegant theory free of artificial restrictions. A more natural class may be obtained as follows. Let $\psi$ be a solution of the differential equation

$$
\mathfrak{Y} \psi+c \psi=0 .
$$

In any interval not containing a zero of $\psi$ we may define a new operator $\mathfrak{A}_{\psi}$ by

$$
\mathfrak{A}_{\psi} f=\frac{1}{\psi} D_{m} \psi^{2} D_{x} \frac{f}{\psi}
$$

${ }^{3}$ A continuous diffusion process in which the total mass is at any time $t$ concentrated in the set of rationals was discussed by Feller and McKean [4]. It is noteworthy that in this case the parabolic partial differential equation $D_{t} u=D_{m} D_{x} u$ is equivalent to an infinite system of ordinary differential equations. 
(precise definitions are postponed to Section 2). It turns out (see Section 5) that

$$
\mathfrak{U}_{\psi} f=\mathfrak{A} f+c f=D_{m} D_{x} f+c f .
$$

Thus $\mathfrak{A}+c$ may be reduced to the form $\mathfrak{A}_{\psi}$, but operators of this form are more general. An arbitrary continuous $\psi>0$ defines an operator $\mathfrak{A}_{\psi}$ (depending on $m$ ). If $\psi$ has a derivative $D_{x} \psi=\psi^{\prime}$, then

$$
\mathfrak{A}_{\psi} f=\frac{1}{\psi} D_{m}\left\{\psi f^{\prime}-\psi^{\prime} f\right\}
$$

implying that each $f$ in the domain of $\mathfrak{A}_{\psi}$ possesses a derivative $D_{x} f=f^{\prime} . \quad$ If $\psi^{\prime}$ is continuously differentiable with respect to $m$, then (1.10) reduces further to $(1.9)$ with

$$
c=-\psi^{-1} \mathfrak{N} \psi=-\psi^{-1} D_{m} D_{x} \psi
$$

If $\psi^{\prime}$ is of bounded variation (without $D_{m} \psi^{\prime}$ existing), the coefficient $c$ can no longer be expressed as a function, but an integrated version of (1.9) remains valid: for each open subinterval

$$
\int_{x_{1}}^{x_{2}}\left(\mathfrak{A}_{\psi} f\right) d m=\left.D_{x} f\right|_{x_{1}} ^{x_{2}}-\int_{x_{1}}^{x_{2}} \psi^{-1} f \cdot d \psi^{\prime} .
$$

It is thus seen that operators of the form (1.8) give a meaning to the notion of $\mathfrak{Y} f+c f$ for the case where $c$ is, say, a Dirac function. ${ }^{4}$ They permit us to treat, for example, a vibrating string with an elastic force acting at a single point just as if the coefficients were continuous. However, the operators $\mathfrak{A}_{\psi}$ are more general than the integrated version (1.12) of $D_{m} D_{x}+c$, and more easy to treat than $D_{m} D_{x}+c$.

After these explanatory remarks we may describe the main contents of this paper as follows. Suppose we are given a (not necessarily finite) interval $I:\left(\beta_{1}, \beta_{2}\right)$ and in it a strictly increasing function $m$ (i.e. a positive measure), not necessarily bounded at the endpoints $\beta_{i}$. Furthermore, we suppose given in $I$ a continuous function $\psi>0$. With these data we define an operator

${ }^{4}$ Such is the case for $\psi$ defined by $2 \psi(x)=1+|x|$. As an illustrative example consider the operator $\mathfrak{A}_{\psi}$ with this $\psi$ and $m(x)=x(-\infty<x<\infty)$. A trivial calculation shows that $\mathfrak{A}_{\psi} f(x)=D_{x}^{2} f(x)$ for each $x \neq 0$. At the origin both $f$ and $D_{x}\left(f \psi^{-1}\right)$ must be continuous, i.e. for each $f$ in the domain of $\mathfrak{A}_{\psi}$ :

$$
\begin{aligned}
\mathfrak{A}_{\psi} f(x) & =f^{\prime \prime}(x), \\
f^{+}(0)-f^{-}(0) & =f(0),
\end{aligned}
$$

while the value of $\mathfrak{A}_{\psi} f$ at the origin is defined by continuity. Now (**) is equivalent to (1.12) with the Dirac function at the origin: the integral on the left equals $f^{\prime}\left(x_{2}\right)-f^{\prime}\left(x_{1}\right)$ if $\left(x_{1}, x_{2}\right)$ does not include the origin, but equals $f^{\prime}\left(x_{2}\right)-f^{\prime}\left(x_{1}\right)-f(0)$ if $x_{1}<0<x_{2}$. Observe that $\mathfrak{A}_{\psi}$ in no way involves discontinuous functions, and that its theory is trivially reducible to special operators of the form $D_{m} D_{x}$. 
$\mathfrak{A}_{\psi}$ as in (1.8). Observe that $\psi$ is a solution of the differential equation $\mathfrak{N}_{\psi} \phi=0$. The most general solution of this equation is defined by

$$
\phi(x)=k_{1} \psi(x) \int_{x_{0}}^{x} \psi^{-2}(s) d s+k_{2} \psi(x),
$$

where $k_{1}$ and $k_{2}$ are constants. Such a $\phi$ can have at most one zero, and in any interval not containing it, we have

$$
\mathfrak{U}_{\phi}=\mathfrak{U}_{\psi} \text {. }
$$

In other words, our operator is completely determined by the interval $I$, the measure $m$, and the two-parametric family of functions annihilated by it. The representation in the form (1.8) is therefore essentially unique.

Operators $\mathfrak{A}_{\psi}$ of this sort are a natural generalization ${ }^{5}$ of the familiar second order differential operators. It is the purpose of this paper to show that the classical Sturm-Liouville and other theories carry over in a natural way leading to a theory which is simpler and more uniform.

Before reviewing the main results of the paper we remark that the general operators $\mathfrak{A}_{\psi}$ are by no means more complicated than the operators $\mathfrak{A}$ of the special form (1.4). In fact (see Section 4), each operator $\mathfrak{A}_{\psi}$ can be mapped onto a "related" operator $D_{\mu} D_{\xi}$ in such a way that no special theory is required. For all intents and purposes it suffices therefore to study operators of the special form (1.4). Their analysis simplifies greatly in consequence of their pleasing property that $\mathfrak{U} f>0$ implies the convexity of $f$.

In Section 3 we introduce a boundary classification. The boundary points which we call active correspond to the Grenzkreis type in $\mathrm{H}$. Weyl's now classical disjunction (see [7]). However, we subdivide Weyl's Grenzpunkt type by introducing the notion of semiactive boundaries. This subclass is introduced for the discussion of bounded solutions and has no bearing or meaning when the Hilbert space norm (1.5) is adopted (which explains why

${ }^{5}$ The operator $\ell=a D_{x}^{2}+b D_{x}+c, a>0$, has the two obvious properties: (1) it is of local character, (2) the minimum property: if $f$ and $\& f$ are continuous in a neighborhood of $x_{0}$ and $f \geqq 0, f\left(x_{0}\right)=0$, then $\& f\left(x_{0}\right) \geqq 0$. It has been shown that the most general operator with these properties has the form of $\mathfrak{A}_{\psi}$ except for the possible presence of singular points at which $\&$ reduces to a first order operator. If condition (2) is replaced by the stronger requirement that at a place of a local minimum $x_{0}$ always $\& f\left(x_{0}\right) \geqq 0$ (i.e. if the hypothesis $f\left(x_{0}\right)$ be deleted), then $\mathfrak{A}_{\psi}$ is of the special form $\mathfrak{A}$ of (1.4). (Cf. [1] and [2].)

A point $x_{0}$ is nonsingular if the equation $\Omega_{\phi}=0$ admits near $x_{0}$ of two independent solutions. If $\psi$ is such a solution and $\psi\left(x_{0}\right)=0$, the right side in (1.13) defines $\phi(x)$ near and at $x_{0}$ by continuity. If $\psi$ has two or more zeros, so does each solution $\phi$ of $\Re_{\phi}=0$, but the connection (1.13) remains valid.

The condition that $\psi>0$ introduced in the present paper is a restriction introduced to avoid going into the complex domain. The most general operator $\&$ without singular points is obtained by permitting $\psi$ to have zeros, but requiring that the right side in (1.13) remain meaningful. Near a zero of $\psi$ one has then to use the representation $\mathfrak{R}=\mathfrak{A}_{\phi}$, or use a complex solution $\phi+i \psi$ which is automatically free of zeros. 
it does not occur in the Weyl-Stone theory). It goes without saying that the type of a boundary point is preserved under the above mentioned mapping of $\mathfrak{A}_{\psi}$ onto $D_{\mu} D_{\xi}$.

To continue, consider first the Hilbert space theory with norm (1.5). For each fixed $\lambda$ the simple mapping $f \rightarrow f \psi^{-1}$ carries a pair $f^{*}, F^{*}$ satisfying

$$
\lambda F^{*}-\mathfrak{A}_{\psi} F^{*}=f^{*}
$$

into a pair $f, F$ satisfying

$$
\lambda F-D_{\mu} D_{\xi} F=f,
$$

and the mapping is isometric: $\left\|f^{*}\right\|_{m}=\|f\|_{\mu}$. Accordingly, as far as Hilbert space theory is concerned, it suffices to study the equation (1.16).

In Section 8 it is shown that for each $\lambda>0$ there exists a positivity preserving linear transformation $\mathbb{S}_{\lambda}$ of norm $\leqq 1$ carrying each $f \in \mathrm{H}_{m}$ into a S $_{\lambda} f=F \epsilon \mathrm{H}_{m}$ satisfying (1.16). The solution of (1.16) is unique except when at least one boundary is active. In each case the range $R$ of $\mathcal{G}_{\lambda}$ is independent of $\lambda$; the operators $\mathfrak{S}_{\lambda}$ and $\mathfrak{U} / R$ (the restriction of $\mathfrak{A}$ to $R$ ) are selfadjoint, $\mathfrak{S}_{\lambda}=(\lambda-\mathfrak{U} / R)^{-1}$. (Here $\left.\mathfrak{A}=D_{\mu} D_{\xi}.\right)$

In Section 10 we study arbitrary symmetric bounded linear transformations $\left.{ }^{3}\right\rangle_{\lambda}$ such that $F=B_{\lambda} f$ satisfies (1.16) for each $f \epsilon \mathbf{H}_{m}$. For a fixed $\lambda$ we describe the most general possible range of such a transformation by appropriate "boundary conditions." Conversely, given a possible range $R$ (i.e. admissible boundary conditions), we show that to each $\lambda>\lambda_{0}$ there corresponds $a \dot{\$}_{\lambda}$ whose range coincides with $R$. Also we find conditions for $\$_{\lambda}$ to be positive or to have norm $\leqq \lambda^{-1}$. Both $\mathfrak{H}_{\lambda}$ and $\mathfrak{A} / R$ are self-adjoint operators. This analysis corresponds to, and amplifies, the well-known discussion in Chapter $\mathrm{X}$ of Stone's treatise [6], but uses simpler methods. (A treatment of unsymmetric operators could proceed following the methods in Section 12.)

New light is shed on the classical boundary conditions in Section 11, where we treat the same problem in the enlarged Hilbert space over the closed interval $\left[\beta_{1}, \beta_{2}\right]$ with norm (1.6). The preceding analysis applies and analogous results hold except that we get a new type of boundary conditions. ${ }^{6}$ On inspection it is seen that they in reality amount to a definition of the operator $\mathfrak{A}$ at the boundary, and that no boundary conditions in the ordinary sense occur as long as the points of the boundary carry a positive weight. The classical boundary conditions are obtained as a degenerate limiting case of this definition of $\mathfrak{A}$ when $m_{i} \rightarrow 0$.

[The Hilbert space theory is contained in Theorem 8.1 and Sections 10, 11. Sections 9, 12-13 have no relation to it, and in the introductory sections all

\footnotetext{
${ }^{6}$ In a disguised form these new boundary conditions occur in the literature as "time dependent" or " $\lambda$-dependent" boundary conditions. They now require no special treatment. The same boundary conditions for continuous and for measure-solutions were discussed in [3].
} 
parts referring to boundedness may be omitted. For the spectral analysis see McKean [5]. ${ }^{7}$ ]

As usual in analysis, the Hilbert space approach leads to the most elegant results. However, our differential operators play an important role in probability theory, the heat conduction, etc., and it is therefore unavoidable to consider them as operators on set functions and on continuous functions. A satisfactory theory can be developed, but new problems arise.

For most purposes it is natural to use the space $\mathbf{C}$ of functions continuous in the closed interval $\bar{I}$ with the traditional norm

$$
\|f\|=\sup _{x \in I}|f(x)| \text {. }
$$

This norm lends itself splendidly for the study of $\mathfrak{A}$, but not equally well for $\mathfrak{A}_{\psi}$. As in the case of the Hilbert space $\mathbf{H}_{m}$, there exists (see Section 8) for each $\lambda>0$ a positive transformation ${ }^{\left(S_{\lambda}\right.}$ from $\mathbf{C}$ to $\mathbf{C}$ such that for each $f \epsilon \mathbf{C}$ the transform $F=\mathcal{S}_{\lambda} f$ has a norm $\|F\| \leqq \lambda^{-1}\|f\|$. However, the mapping which connects (1.15) and (1.16) is no longer norm-preserving. It takes the space $\mathbf{C}$ into the Banach space $\mathbf{C}_{\psi}$ of functions such that $f \psi^{-1}$ is continuous in the closed interval, $\mathbf{C}_{\psi}$ being metrized by the norm

$$
\|f\|_{\psi}=\left\|f \psi^{-1}\right\|=\sup |f(x) \psi(x)| \text {. }
$$

Thus all theorems concerning (1.16) and the classical norm $\|f\|$ translate into equivalent theorems concerning (1.15) and the norm ${ }^{8}$ (1.18). The following theorem (proved in Section 9) is therefore of interest:

In order that the equation (1.15) admit for each $f^{*} \epsilon \mathbf{C}$ a solution $F^{*} \epsilon \mathbf{C}$ such that $\left\|F^{*}\right\| \leqq \lambda\left\|f^{*}\right\|$, it is necessary and sufficient that $\psi$ be convex upward. ${ }^{9}$

By analogy with Sections 10-11 we treat in Section 12 the most general boundary conditions for transformations from $\mathbf{C}$ to $\mathbf{C}$ inverting $\lambda-\mathfrak{A}$. However, while in the case of the Hilbert space norm (1.5) it was natural to restrict the theory to symmetric operators, the requirement of symmetry is meaningless in C. Accordingly, we study arbitrary transformations, and we

${ }^{7}$ McKean considers only Green function transformations and symmetric boundary conditions introduced in [3] and guaranteeing the boundedness of the solutions of (1.16). The mappings of Section 4 automatically extend his results to all transformations from $\mathbf{H}_{m}$ to $\mathbf{H}_{m}$ provided each boundary condition involves only one boundary point (see Note 3 to Theorem 8.1). The newer type boundary conditions applying to the enlarged space $\overline{\mathbf{H}}_{m}$ require only a redefinition of the Green function on the boundary, and thus McKean's analysis could be extended to them by obvious modifications.

${ }^{8}$ Actually several norms are equally applicable to each of the equations (1.15) and (1.16). For example, for $\mathfrak{A}=D_{m} D_{x}$ in the interval $0<x<\infty$ the norm $\|f\|_{x}=$ $\sup \left|f(x) x^{-1}\right|$ is fully as good as (1.17).

${ }^{9} \mathrm{I}$ owe this observation to H. P. McKean, Jr. If $\psi$ is convex upward, so is each positive $\phi$ satisfying $\mathfrak{A}_{\psi} \phi=0$. In the special case of operators $\mathfrak{A}_{\psi}=\mathfrak{A}+c$ the convexity of $\psi$ is equivalent to $c \leqq 0$. 
encounter new "boundary conditions" depending on global functionals, and not only on the boundary.

Section 13 deals with the transformations on measures which are adjoint to the transformations constructed in Section 12. We discover here that in general the adjoint $\mathfrak{U}^{*}$ to $\mathfrak{Y}$ need not be a differential operator. ${ }^{10}$ For $\mathfrak{I}^{*}$ to be of local character it is necessary and sufficient that the boundary conditions for $\mathfrak{A}$ do not involve global functionals, that is, they have to be of the type of the boundary conditions discussed in Section 11 for $\overline{\mathbf{H}}_{m}$. [Sections 12-13 are independent of Sections 10-11.]

It may be observed that the results of Sections 8-13 can be rephrased in the terminology of semigroups. In fact, saying that the transformations ${ }^{S_{\lambda}}$ have, for $\lambda>\lambda_{0}$, a common range $R$ is equivalent to saying that the restriction $\mathfrak{U} / R$ generates a semigroup. ${ }^{11}$

\section{Definitions and preparations}

In a fixed open interval $I:\left(\beta_{1}, \beta_{2}\right)$ (where $\left.-\infty \leqq \beta_{1}<\beta_{2} \leqq \infty\right)$ we suppose given a strictly increasing function $m$, which is continuous to the right: $m(x)=$ $m(x+)$. One should conceive of $m(x)$ as the measure of the half-open interval $\left[\beta_{1}, x\right]$, and the notation using measures and derivatives of measures is really preferable for our purposes, but it seems less popular. We call $m$ the canonical measure for our operators. Needless to say that $m(x)$ is finite for each $x$, but $m$ need not be bounded near the endpoints $\beta_{i}$. (When $\beta_{2}<\infty$, $m\left(\beta_{2}-\right)<\infty$, it is possible, and often desirable, to enlarge the interval $I$ by adding to it the point $\beta_{2}$ carrying a positive weight $m_{2}$.)

If $m$ happens to be continuous, the interval $I$ can be referred to $m$, instead of $x$, as a scale parameter. In this case the right derivative of $f$ with respect to $m$ is defined, as usual, by

$$
D_{m}^{+} f(x)=\lim _{h \downarrow 0} \frac{f(x+h)-f(x)}{m(x+h)-m(x)} .
$$

In the general case we keep this definition at each point of continuity of $m$. (It is clear that at such points $m(x+h)$ in the denominator may be replaced by $m(x+h-)$ without affecting the limit.) At points of discontinuity we

${ }^{10}$ The contents of Sections 12 and 13 are a generalization, but also a great simplification, of the developments in [3] concerning operators of the special type (1.1). Unfortunately, a notational confusion has introduced an error into [3], which is explained below in footnote 20 .

Added in proof. While the present paper was in press the author has received a recent paper [8] by $A . D$. Ventcelj in which the error mentioned above is pointed out and corrected for operators of the special form $D_{m} D_{x}$. In the text we treat lateral conditions for more general operators and also for the case when the Hilbert space norm is used and/or the requirement of positivity is dropped. All results of [3] are superseded by the present paper except that here we refrain from using the terminology of semigroups.

${ }^{11}$ On the generation of semigroups by restrictions of operators and the abstract definition of boundary conditions see [3]. 
replace the denominator by the increment of $m$ over the closed interval $[x, x+h]$. Then

$$
D_{m} f(x)=\frac{f(x+)-f(x-)}{m(x+)-m(x-)}
$$

is not only the right, but also the left, derivative at $x$. For a derivative $D_{m} f$ to exist it is necessary that $f$ be continuous with respect to $m$, jumps being permitted at points of discontinuity of $m$.

As usual $D_{m}$ exists if $D_{m}^{+}=D_{m}^{-}$. The derivative $D_{m}$ is defined by (2.1) and (2.2), respectively, at points of continuity and discontinuity of $m$.

When no confusion can arise, we shall occasionally simplify the notation by inconsistently using the symbol $D_{m}$ in the meaning of: either $D_{m}^{+}$or $D_{m}^{-}$ even if $D_{m}^{+} \neq D_{m}^{-}$or if only one is defined. (Thus it is clear that when $x=\beta_{2}$ is substituted in $D_{m} f$ we should read $D_{m}^{-} f\left(\beta_{2}\right)$.)

We agree that in some interval $I_{0}$ the function $f$ can belong to the domain of the operator $D_{m} D_{x}$ only if the one-sided derivatives $f^{+}=D_{x}^{+} f$ and $f^{-}=D_{x}^{-} f$ exist everywhere and are continuous with respect to $m$. The value of $D_{m} D_{x} f$ is then defined at each point where the derivative exists. The definition of $\mathfrak{A}=D_{m} D_{x}$ will be completed by specifying the domain: in connection with the space $\mathbf{C}$ of continuous functions we shall require that $D_{m} D_{x} f \epsilon \mathbf{C}$; when the Hilbert space norm (1.5) is used, we shall naturally require that $F=D_{x} f$ be absolutely continuous with respect to $m$ and $\left\|D_{m} F\right\|_{m}<\infty$. Under any circumstances

$$
\int_{\alpha+}^{\beta-} \mathfrak{A} f \cdot d m=f^{-}(\beta)-f^{+}(\alpha) .
$$

Note. It is best to conceive of $f^{\prime}$, which is a function of bounded variation, as inducing a signed measure. The operator $D_{x}$ then changes point functions into set functions, and $D_{m}$ (a Radon-Nikodym derivative) changes the measure back into a point function. The formal adjoint $D_{x} D_{m}$ operates on set functions, and $D_{m}$ maps a set function into a point function.

Coming to notations it will be noticed that, as usual, 0 and $x$ denote not only numbers, but also the obvious functions. However, to avoid confusion, we shall use the symbol 1 to denote the function which equals 1 everywhere.

Existence of solutions. Let $c$ be a real function such that

$$
\int_{I_{0}}|c| d m<\infty
$$

for each subinterval $I_{0}$ of $I$. For arbitrarily prescribed constants $k_{1}, k_{2}$ the classical method of successive approximations shows that there exists a unique function $u$ defined throughout $I$ and satisfying almost everywhere $(m)$ the differential equation

$$
D_{m} D_{x} u=c u
$$

with the initial conditions 


$$
u(\beta)=k_{1}, \quad u^{+}(\beta)=k_{2} .
$$

More precisely,

$$
u(x)=k_{1}+k_{2} \not t+\int_{\beta}^{x} d s \int_{\beta}^{s} c u d m .
$$

In fact, putting $u^{(0)}(x)=k_{1}+k_{2}(x-\beta)$ and

$$
u^{(n+1)}(x)=k_{1}+k_{2}(x-\beta)+\int_{\beta}^{x} d s \int_{\beta}^{s} c u^{(n)} d m
$$

it is seen by induction that

$$
\begin{aligned}
\left|u^{(n+1)}(x)-u^{(n)}(x)\right| & \leqq \int_{\beta}^{x} d s \int_{\beta}^{s}|c| \cdot\left|u^{(n)}-u^{(n-1)}\right| \cdot d m \\
& \leqq K \cdot q^{n}
\end{aligned}
$$

provided $|x-\beta|<\delta$ where $\delta$ is chosen so that

$$
2 \delta \int_{\beta-\delta}^{\beta+\delta}|c| d m<q<1 .
$$

It follows that $u^{(n)}$ converges in the interval $(\beta-\delta, \beta+\delta)$ uniformly to a solution of (2.6), and the argument shows at the same time that $u=0$ is the only solution when $k_{1}=k_{2}=0$. This solution $u$ can be continued by the same procedure to an arbitrary subinterval of $I$.

\section{Minimal solutions. Classification of boundaries}

Consider the operator $\mathfrak{A}_{\psi}$ defined by (1.8) where $\psi>0$ is continuous in $I:\left(\beta_{1}, \beta_{2}\right)$. A function $\phi$ satisfies $\mathfrak{A}_{\psi} \phi=0$ if, and only if,

$$
\psi^{2}(\phi / \psi)^{\prime}=\text { const. }
$$

Note that this relationship is really symmetric in $\phi$ and $\psi$ since in each interval not containing a zero of $\phi$

$$
\psi^{2}(\phi / \psi)^{\prime}=-\phi^{2}(\psi / \phi)^{\prime} .
$$

The ratio $\phi / \psi$ is necessarily monotonic, and hence $\phi$ can have at most one zero.

Lemma 3.1. Among the functions $\phi$ satisfying (3.1) (or, what amounts to the same, (1.13)) there exists one, $\phi_{*}$, such that

$$
\int_{\beta}^{\beta_{2}} \phi_{*}^{-2}(s) d s=\infty
$$

for each $\beta \in I$. Each solution $\phi$ of (3.1) which is not a multiple of $\phi_{*}$ satisfies

$$
\int_{\beta}^{\beta_{2}} \phi^{-2}(s) d s<\infty, \quad \frac{\phi_{*}(x)}{\phi(x)} \rightarrow 0 \quad \text { as } \quad x \rightarrow \beta_{2}
$$

provided $\phi(x) \neq 0$ for $x>\beta$. 
We shall say that $\phi_{*}$ is minimal at $\beta_{2}$. The solution which is minimal at $\beta_{1}$ is defined in like manner.

Proof. Let $\phi$ be a solution of (3.1) which is independent of $\psi$. It is seen from (1.13) that as $x \rightarrow \beta_{2}$

$$
\left|\phi(x) \psi^{-1}(x)\right| \rightarrow \infty \quad \text { if, and only if, } \quad \int_{\beta}^{\beta_{2}} \psi^{-2}(s) d s=\infty
$$

for some (and therefore for each) $\beta \epsilon I$. Because of symmetry this statement holds also when $\phi$ and $\psi$ are interchanged. Since it is impossible that both $\phi \psi^{-1}$ and $\psi \phi^{-1}$ tend to $\pm \infty$, it follows that the minimal solution, if it exists, is determined up to a constant multiplier. On the other hand, if the integral in (3.5) converges, put

$$
\phi_{*}(x)=\psi(x) \int_{x}^{\beta_{2}} \psi^{-2}(s) d s .
$$

Then $\psi(x) \phi_{*}^{-1}(x) \rightarrow \infty$ as $x \rightarrow \beta_{2}$, and therefore (3.3) holds. Thus either $\psi$ or the solution defined by (3.6) is minimal. The lemma is proved.

(Note that $\psi$ may be minimal both at $\beta_{1}$ and $\beta_{2}$. In this case for each solution $\phi$ of (3.1) which is independent of $\psi$, the ratio $\phi / \psi$ is monotonic and goes from $-\infty$ to $\infty$. On the other hand, if the solutions $\phi_{* 1}$ and $\phi_{* 2}$ which are minimal at $\beta_{1}$ and $\beta_{2}$, respectively, are independent, both may be taken as positive, and there exist then infinitely many positive solutions of (3.1).)

Definition 3.1. Let $\psi>0$ be continuous in I. A solution $\phi_{*}$ of $\mathfrak{A}_{\psi} \phi_{*}=0$ will be called minimal at $\beta_{2}$ if (3.3) holds.

Definition 3.2. Let $\phi_{*}$ be minimal for $\mathfrak{A}_{\psi}$ at $\beta_{2}$, and let $\phi$ be any solution of $\mathfrak{A}_{\psi} \phi=0$ which is independent of $\phi_{*}$.

The endpoint $\beta_{2}$ will be called active for $\mathfrak{A}_{\psi}$ if for some (and consequently for each) $\beta \in I$

$$
\int_{\beta}^{\beta_{2}} \phi^{2} d m<\infty .
$$

$A$ nonactive endpoint $\beta_{2}$ will be called semiactive if

$$
\int_{\beta}^{\beta_{2}}\left|\phi \phi_{*}\right| d m<\infty \text {. }
$$

Each solution being a linear combination of $\phi$ and $\phi_{*}$, it is clear that this definition does not depend on the choice of $\phi$. The definition applies to the left boundary with the integrals extended over $\left(\beta_{1}, \beta\right)$.

In the case of the simple operator $\mathfrak{A}=D_{m} D_{x}$ we can put

$$
\begin{array}{lll}
\phi_{*}(x)=\beta_{2}-x, & \phi(x)=1 & \text { if } \quad \beta_{2}<\infty \\
\phi_{*}(x)=1, & \phi(x)=x & \text { if } \quad \beta_{2}=\infty .
\end{array}
$$

The endpoint $\beta_{2}$ is active for $\mathfrak{A}=D_{m} D_{x}$ if, and only if, 


$$
\int_{\beta}^{\beta_{2}} x^{2} d m(x)<\infty .
$$

It is semiactive if the integral in (3.10) diverges but

$$
\begin{array}{cc}
\int_{\beta}^{\beta_{2}}\left(\beta_{2}-x\right) d m(x)<\infty & \text { in case } \beta_{2}<\infty \\
\int_{\beta}^{\beta_{2}} x d m(x)<\infty & \text { in case } \beta_{2}=\infty .
\end{array}
$$

Obviously the first inequality in (3.11) is equivalent $\mathrm{o}^{12}$

$$
\int_{\beta}^{\beta_{2}} m(y) d y<\infty \quad \beta_{2}<\infty .
$$

\section{Related operators}

Let $\psi>0$ be continuous in $I:\left(\beta_{1}, \beta_{2}\right)$. For a fixed $\beta \epsilon I$ define a function $\xi$ by

$$
\xi(x)=\int_{\beta}^{x} \psi^{-2}(s) d s .
$$

This maps $I$ onto an interval $I^{*}:\left(\beta_{1}^{*}, \beta_{2}^{*}\right)$, and in it we define a monotone right-continuous function $\mu(\xi)$ by

$$
\mu(\xi)=\int_{\beta}^{x} \psi^{2}(s) d m(s)
$$

where $x$ stands for the point mapped on $\xi$. In $I^{*}$ we define the operator

$$
\mathfrak{A}^{*}=D_{\mu} D_{\xi} .
$$

We shall say that $(\mathfrak{A}, I)$ and $\left(\mathfrak{H}^{*}, I^{*}\right)$ are related by the transformation (4.1) (4.2). Needless to say that this term will be employed also if $\xi$ is replaced by a linear function $k_{1} \xi+k_{2}$, say, $-\xi$ (cf. (4.9)).

${ }^{12}$ In [3] a classification of boundaries was introduced for operators of the special type $a D_{x}^{2}+b D_{x}$. It was intended for the study of boundedness and/or integrability of solutions, and did not pay attention to the Hilbert space norm (1.5). This classification may be carried over to our more general operators $\mathfrak{A}=D_{m} D_{x}$. It is based only on the criteria (3.11) and (3.12), and according to it $\beta_{2}$ is for $\mathfrak{A}$

regular if $\boldsymbol{\beta}_{2}<\infty, m\left(\boldsymbol{\beta}_{2}-\right)<\infty$;

exit if not regular, $\beta_{2}<\infty$, and (3.11) holds;

entrance if not regular, $\beta_{2}=\infty$, and (3.12) holds;

natural otherwise.

Thus:

$$
\begin{aligned}
& \text { an active } \beta_{2} \text { is } \begin{cases}\text { regular } & \text { if } \beta_{2}<\infty, m\left(\beta_{2}-\right)<\infty \\
\text { exit } & \text { if } \beta_{2}<\infty, m\left(\beta_{2}-\right)=\infty \\
\text { entrance } & \text { if } \beta_{2}=\infty\end{cases} \\
& \text { a semiactive } \beta_{2} \text { is } \begin{cases}\text { exit } & \text { if } \beta_{2}<\infty \\
\text { entrance if } \beta_{2}=\infty .\end{cases}
\end{aligned}
$$

A $\beta_{2}$ which is neither active nor semiactive is natural, and conversely. 
To each function $f$ continuous on $I$ we let correspond a function $f^{*}$ defined on $I^{*}$ by means of the inverse mapping to (4.1) as $f^{*}=f \psi^{-1}$, i.e. by

$$
f^{*}(\xi)=f(x(\xi)) \cdot \psi^{-1}(x(\xi)) .
$$

Clearly

$$
\mathfrak{A}_{\psi}=\psi \cdot \mathfrak{2} *
$$

in the obvious sense. The following theorem is now trivially verified:

Theorem 4.1. For each $f$

$$
\int_{I^{*}}\left|f^{*}\right|^{2} d \mu=\int_{I} f^{2} d m
$$

If $\lambda F-\mathfrak{A}_{\psi} F=f$, then $\lambda F^{*}-\mathfrak{A}^{*} F^{*}=f^{*}$. With the norms introduced by (1.17), (1.18) we have

$$
\left\|f^{*}\right\|=\|f\|_{\psi} .
$$

In particular, $\psi^{*}=1$ and $\mathfrak{A}_{\psi} \phi=0$ implies $\mathfrak{A}^{*} \phi^{*}=0$. Recalling that $\phi$ is minimal for $\mathfrak{A}_{\psi}$ at $\beta_{2}$ if, and only if, for each solution $\phi_{1}$ of $\mathfrak{A}_{\psi} \phi_{1}=0$ which is independent of $\phi$ the ratio $\phi(x) \phi_{1}^{-1}(x) \rightarrow 0$ as $x \rightarrow \beta_{2}$, we get

Lemma 4.1. The mapping $f \rightarrow f^{*}$ carries solutions of $\mathfrak{A}_{\psi} \phi=0$ into solutions of $\mathfrak{H}^{*} \phi^{*}=0$, and the property of minimality is preserved.

The type of the boundary $\beta_{2}$ for $\mathfrak{A}_{\psi}$ depends on the integral (3.7), i.e. on the behavior of $m$ near the endpoint $\beta_{2}$. To investigate the relationship between the types of $\beta_{2}$ and $\beta_{2}^{*}$, we must therefore replace the interval $I$ by a subinterval $\left(\beta, \beta_{2}\right)$ and apply the mapping to it. The convergence of all integrals occurring in the criterion then depends only on a vicinity of the right endpoint, and (4.6) together with Lemma 4.1 show directly the truth of

Theorem 4.2. The endpoint $\beta_{i}$ is for $\mathfrak{A}_{\psi}$ of the same type (active, semiactive, or neither) as the endpoint $\beta_{i}^{*}$ is for $\mathfrak{2}^{*}$.

Note that $\mathfrak{A}_{\psi} \phi=0$ may admit of several positive solutions in which case $\mathfrak{A}_{\psi}=\mathfrak{A}_{\phi}$ (see Theorem 5.1). Thus $\mathfrak{A}_{\psi}$ may be mapped in several ways on operators of the simpler type $D_{\mu} D_{\xi}$. (Under any circumstances different such mappings are available in each proper subinterval of $I$, and in particular, for neighborhoods of the endpoints.) For example, in $(0, \infty)$

$$
D_{m} D_{x} f=x^{-1} D_{m} x^{2} D_{x}\left(f x^{-1}\right) .
$$

Thus the operator $\mathfrak{A}=D_{m} D_{x}$ in $(0, \infty)$ is related to the operator of the same type $\mathfrak{2}^{*}=D_{\mu} D_{\xi}$ by means of

$$
\xi=x^{-1}, \quad \mu(\xi)=-\int_{1}^{\xi-1} s^{2} d m(s)
$$


Here $f^{*}(\xi)=f\left(\xi^{-1}\right) \xi$, and the endpoint $\infty$ corresponds to 0 . This transformation enables us always to reduce the study of the behavior of solutions near a boundary $\beta_{2}=\infty$ to the neighborhood ${ }^{13}$ of 0 .

\section{A basic identity and its corollaries}

We suppose that in the interval $I$ the monotonic function $m$ and the continuous $\psi>0$ are given; we consider the operator $\mathfrak{A}_{\psi}$ defined by (1.8) and other operators of the same type obtained by varying $\psi$ while keeping $m$ fixed.

LEMmA 5.1. Let $U>0$ and $V$ be functions such that $\mathfrak{A}_{\psi} U$ and $\mathfrak{A}_{\psi} V$ exist and are continuous in $I$. Then

$$
U \cdot \mathfrak{A}_{\psi} V-V \cdot \mathfrak{A}_{\psi} U=U \cdot \mathfrak{A}_{U} V .
$$

Proof. For each subinterval $(\alpha, \beta)$ of $I$ we have

$$
\int_{\alpha}^{\beta} U \cdot \mathfrak{A}_{\psi} V \cdot d m=\int_{\alpha}^{\beta} U \psi^{-1} \cdot d\left\{\psi^{2}(V / \psi)^{\prime}\right\}
$$

where the prime indicates differentiation with respect to the variable of integration. A simple integration by parts leads hence to

$$
\begin{aligned}
\int_{\alpha}^{\beta}\left\{U \cdot \mathfrak{A}_{\psi} V-V \cdot \mathfrak{A}_{\psi} U\right\} \cdot d m & =\left.\psi^{2} \cdot\left\{\frac{U}{\psi}\left(\frac{V}{\psi}\right)^{\prime}-\frac{V}{\psi}\left(\frac{U}{\psi}\right)^{\prime}\right\}\right|_{\alpha} ^{\beta} \\
& =\left.U^{2} \cdot\left(\frac{V / \psi}{U / \psi}\right)^{\prime}\right|_{\alpha} ^{\beta}=\left.U^{2} \cdot\left(\frac{V}{U}\right)^{\prime}\right|_{\alpha} ^{\beta} .
\end{aligned}
$$

This is the integrated version of (5.1), and the lemma is proved.

TheOREM 5.1. If $\phi$ is a positive solution of $\mathfrak{A}_{\psi} \phi=0$, then

$$
\mathfrak{P}_{\psi}=\mathfrak{U}_{\phi} \text {. }
$$

(That is to say, the representation (1.8) has an intrinsic character, independent of the choice of the null-solution $\psi$.)

Proof. Set $U=\phi$ in (5.1).

LEMma 5.2. We have

$$
\mathfrak{A}_{\psi} V-c V=\mathfrak{A}_{U} V \quad \text { where } c=U^{-1} \cdot \mathfrak{A}_{\psi} U \text {. }
$$

In particular: given an arbitrary integrable function $c$, in the neighborhood of any point it is possible to reduce the operator $\mathfrak{A}_{\psi}-c$ to the form $\mathfrak{A}_{U}$ involving the same canonical measure $m$.

\footnotetext{
${ }^{13}$ In the terminology of [3] explained in the preceding footnote this means that an exit boundary may be transformed into an entrance boundary, and vice versa. A theorem such as "at an exit boundary a solution of $\lambda u-\mathfrak{A} u=0$ approaches a finite limit" is equivalent to "at an entrance boundary the ratio $u(x) / x$ approaches a finite limit" etc. This may serve to explain the formal advantages and simplicity of the present approach.
} 
Proof. Divide (5.1) by. $U$ to obtain (5.5). We are free to choose $U$ as a solution of $\mathfrak{A}_{\psi} U-c U=0$ (see Section 2 concerning its existence).

Lemma 5.3. (Wronski identity). If $\lambda u_{i}-\mathfrak{A}_{\psi} u_{i}=0$, where $i=1,2$ and $\lambda$ is a constant, then

$$
u_{1}^{2}\left(u_{2} / u_{1}\right)^{\prime}=\text { const. }
$$

In particular, $\lambda u_{i}-D_{m} D_{x} u_{i}=0$ implies

$$
u_{1} u_{2}^{\prime}-u_{2} u_{1}^{\prime}=\text { const. }
$$

Proof. Clearly (5.6) is a special case of (5.3). When $u_{1}$ and $u_{2}$ are differentiable with respect to $x$, then (5.6) reduces to (5.7). [Note that (5.7) holds both for the right and the left derivative.]

\section{The homogeneous equation}

For simplicity of exposition we first consider the reduced operator $\mathfrak{A}$ of (1.4) in an interval $I:-\infty \leqq \beta_{1}<x<\beta_{2} \leqq \infty$. We are interested in solutions $u$ of the equation

$$
\lambda u-D_{m} u^{\prime}=0, \quad \lambda>0
$$

defined throughout $I$; here $\lambda$ is a constant.

For an arbitrary point $\beta \epsilon I$ there exists (see Section 2) a solution $u$ satisfying the initial conditions $u(\beta)=1, u^{+}(\beta)=0$. Remembering that $D_{m} u^{\prime}>0$ implies that $u^{\prime}$ increases, i.e. that $u$ is convex, we see that $u \geqq 1$. It follows from Lemma 5.2 that the operator $\lambda-D_{m} D_{x}$ is identical with the reduced operator $\mathfrak{A}_{u}$, and we can apply Lemma 3.1 to conclude that there exist solutions $u_{* 1}$ and $u_{* 2}$ of (6.1) which are minimal, respectively, at $\beta_{1}$ and $\beta_{2}$. For each $\beta \epsilon I$ we have then

$$
\int_{\beta_{1}}^{\beta} u_{* 1}^{-2}(x) d x=\infty, \quad \int_{\beta}^{\beta_{2}} u_{* 2}^{-2}(x) d x=\infty .
$$

Near $\beta_{2}$ the solution $u_{* 2}$ is positive, and hence convex. If it were increasing, there should exist an $\varepsilon>0$ such that $u_{* 2}(x)>\varepsilon(x-\alpha)$ for $x>\alpha$, which contradicts the condition that the second integral in (6.2) diverges for each $\beta$. Thus $u_{* 2}$ is positive and decreasing near $\beta_{2}$; this implies that $u_{* 2}$ is convex and decreasing throughout $I$. We have thus

THEOREM 6.1. For each $\lambda>0$ the equation $\lambda u-\mathfrak{A} u=0$ admits of two positive convex solutions $u_{* 1}$ and $u_{* 2}$ such that $u_{* 1} \uparrow$ while $u_{* 2} \downarrow$ throughout the interval I, and that (6.2) holds.

(This condition determines the solutions up to a multiplicative constant. We shall say that $u_{* i}$ is minimal at $\beta_{i}$.)

The remaining part of this section is devoted to a study of the solutions near $\beta_{2}$, and the interval $\left(\beta_{1}, \beta_{2}\right)$ may be replaced by an arbitrary subinterval 
$\left(\beta, \beta_{2}\right)$. Needless to say that the left boundary is subject to the same analysis. Integrate (6.1) to get

$$
u^{+}(x)=u^{+}(\beta)+\int_{\beta+}^{x+} \lambda u d m, \quad \beta<x .
$$

For a positive, decreasing $u$ the left side is $<0$ while the integrand is $>0$. Accordingly $-u^{+}(\beta)$ is an upper bound for the integral for all $x>\beta$. We have thus

LEMma 6.1. For each positive decreasing solution $u$ of (6.1) and each $\beta \in I$

$$
\int_{\beta}^{\beta 2} u d m<\infty
$$

This lemma applies, in particular, to the solution $u_{*}$ minimal at $\beta_{2}$ and will be used prominently in the next section.

For the reader's convenience we shall first take up the case $\beta_{2}<\infty$ and reduce the case $\beta_{2}=\infty$ to it. However, this separation is by no means necessary, and the case $\beta_{2}=\infty$ can be treated directly by means of the simple estimates here used for $\beta_{2}<\infty$.

Consider again the solution $u$ of (6.1) satisfying the initial conditions $u(\beta)=1, u^{+}(\beta)=0$. It is obvious from $(6.3)$ that $u^{+}(x)>\lambda\{m(x)-m(\beta)\}$ for each $x>\beta$. Therefore $u^{+}$can be integrable over $\left(\beta, \beta_{2}\right)$ only if

$$
\infty>\int_{\beta}^{\beta_{2}}\{m(x)-m(\beta)\} d x=\int_{\beta}^{\beta_{2}}\left(\beta_{2}-y\right) d m(y) .
$$

On the other hand, (6.3) shows that

$$
u^{+}(x)<\lambda u(x)\{m(x)-m(\beta)\},
$$$$
x>\beta,
$$

and therefore (6.5) implies that $\log u$ has an integrable derivative. In other words, $u$ remains bounded at $\beta_{2}$ if, and only if, (6.5) holds. Remembering that every solution of (6.1) is a linear combination of $u$ and $u_{* 2}$ we see that in this case all solutions of (6.1) remain bounded at $\beta_{2}$.

Suppose now that $m$ remains bounded at $\beta_{2}$, that is, suppose $\beta_{2}<\infty$ and $m\left(\beta_{2}-\right)<\infty$. Then (6.5) holds, and each solution $u$ remains bounded. Moreover, in view of (6.3) the derivative $u^{+}(x)$ approaches a finite limit $u^{-}\left(\beta_{2}\right)$ as $x \rightarrow \beta_{2}$. In this case the boundary point $\beta_{2}$ may be treated just as an interior point, and the classical method of successive approximations (see Section 2) shows that to arbitrary constants $A, B$ there exists exactly one solution of (6.1) such that

$$
u\left(\beta_{2}\right)=A, \quad u^{-}\left(\beta_{2}\right)=B .
$$

In particular, $u_{* 2}\left(\beta_{2}\right)=0$ and $u_{*_{2}}\left(\beta_{2}\right)<0$.

Next suppose that $m\left(\beta_{2}-\right)=\infty$ but that (6.5) holds. We have seen that in this case all solutions $u$ remain bounded at $\beta_{2}$. From (6.3) we see that $u^{+}(x) \rightarrow \infty$ for each increasing $u$, and therefore no solution $u$ independent of 
$u_{* 2}$ has a bounded derivative; in particular, there does not exist a positive decreasing solution independent of $u_{* 2}$. It is clear from (6.4) that $u_{* 2}\left(\beta_{2}\right)=0$. For the sake of completeness we show that $u \bar{*}_{2}\left(\beta_{2}\right)<0$. Because of the convexity of $u_{* 2}$ we have $u_{* 2}(s)\left(\beta_{2}-s\right)^{-1}<-u_{* 2}^{+}(s)<-u_{*_{2}}^{+}(\beta)$ for each $s>\beta$, and hence we conclude from (6.3) that

$$
-u_{* 2}^{+}(x)>-u_{*_{2}}^{+}(\beta)\left\{1-\lambda \int_{\beta}^{x}\left(\beta_{2}-s\right) d m(s)\right\} .
$$

We may choose $\beta$ so close to $\beta_{2}$ that the integral in (6.5) is smaller than $(2 \lambda)^{-1}$. Then $-u_{* 2}^{+}(x)>-\frac{1}{2} u_{*_{2}}^{+}(\beta)$ for each $x>\beta$, and hence

as asserted.

$$
-\bar{*}_{2}\left(\beta_{2}\right) \geqq-\frac{1}{2} u_{*}^{+}(\beta)>0,
$$

Finally, when (6.5) does not hold, each solution $u$ independent of $u_{* 2}$ is unbounded at $\beta_{2}$, while (6.4) implies that $u_{* 2}\left(\beta_{2}\right)=u \bar{w}_{2}\left(\beta_{2}\right)=0$.

Recalling Definition 3.2 we can summarize these findings in the following theorem (only part (i) has a bearing on the Hilbert space approach).

Theorem 6.2. Let $\beta_{2}<\infty$.

(i) If $\beta_{2}$ is active (that is, if $m\left(\beta_{2}-\right)<\infty$ ), each solution $u$ of (6.1) and its derivative $u^{+}$approach finite limits at $\beta_{2}$. Exactly one $u$ satisfies the terminal condition (6.7), and hence there exist independent positive decreasing solutions. For the minimal $u_{*}$ we have $u_{*}\left(\beta_{2}\right)=0$ and $\overline{u_{*}}\left(\beta_{2}\right) \neq 0$.

(ii) If $\beta_{2}$ is semiactive (that is, $m\left(\beta_{2}-\right)=\infty$ but (6.5) holds), each $u$ is bounded at $\beta_{2}$, but $\left|u^{\prime}(x)\right| \rightarrow \infty$ as $x \rightarrow \beta_{2}$ except if $u$ is a multiple of $u_{*}$. The latter is the only positive decreasing solution and $u_{*}\left(\beta_{2}\right)=0,0>u_{*}\left(\beta_{2}\right)>-\infty$.

(iii) If $\beta_{2}$ is neither active nor semiactive, then $u_{*}\left(\beta_{2}\right)=u \bar{*}\left(\beta_{2}\right)=0$. Each $u$ independent of $u_{*}$ is unbounded.

It has been pointed out in Section 4 that the transformation (4.9) permits us to reduce the case $\beta_{2}=\infty$ to the case $\beta_{2}<\infty$. Without loss of generality we may suppose $\beta_{1}<0<\beta_{2}=\infty$. Then (4.9) carries the boundary point $\infty$ for $D_{m} D_{x}$ into the left endpoint 0 for $D_{\mu} D_{\xi}$, and the type of the boundary does not change. There exists in $(0, \infty)$ a one-to-one correspondence between the solutions $u$ of $\lambda u-D_{m} D_{x} u=0$ and the solutions $u^{*}$ of $\lambda u^{*}-D_{\mu} D_{\xi} u^{*}=0$ established by the relation $u^{*}(\xi)=\xi u\left(\xi^{-1}\right)$. Applying the last theorem to $u^{*}$ and reading it in terms of $u$ we have

Theorem 6.3. Let $\beta_{2}=\infty$.

(i) If $\infty$ is an active boundary (that is, if $\int^{\infty} x^{2} d m<\infty$ ), then for each solution $u$ of $(6.1)$

$$
u(x)=A x+B+o(1)
$$$$
x \rightarrow \infty
$$

where $A$ and $B$ are constants. To prescribed $A, B$ there exists exactly one solution $u$ satisfying the terminal condition (6.9). The minimal solution $u_{*} \downarrow B>0$.

(ii) If $\infty$ is semiactive (i.e. $\int^{\infty} x^{2} d m=\infty$ but $\left.\int^{\infty} x d m<\infty\right)$, then for each solution $u$ which is near $\infty$ increasing 


$$
x^{-1} u(x) \rightarrow A<\infty, \quad \text { but } \quad A x-u(x) \rightarrow \infty \text { as } x \rightarrow \infty .
$$

The minimal solution $u_{*} \downarrow B>0$, and no solution independent of $u_{*}$ is bounded at $\infty$.

(iii) If $\int^{\infty} x d m=\infty$, then $u_{*} \downarrow 0$. For each solution $u$ independent of $u_{*}$ we have $x^{-1} u(x) \rightarrow \pm \infty$.

In reality the last two theorems can be formulated jointly using the notation (3.9). With this notation, however, the formulation applies at once to the more general operator $\mathfrak{A}_{\psi}$ of (1.8). A glance at Theorem 4.1 and Lemma 4.1 will show that the last two theorems are a special case of the following (which, for brevity, we state explicitly only for active boundaries).

Theorem 6.4. The equation

$$
\lambda u-\psi^{-1} D_{m}\left(\psi^{2} D_{x}\left(u \psi^{-1}\right)\right)=0, \quad \lambda>0
$$

admits of two independent positive solutions $u_{* i}$ such that (6.2) holds.

Let $\phi$ be a solution of $\mathfrak{A}_{\psi} \phi=0$ which is at $\beta_{2}$ positive but not minimal, and let $\phi_{*}$ be the solution of $\mathfrak{H}_{\psi} \phi_{*}=0$ which is minimal at $\beta_{2}$. Then:

If $\beta_{2}$ is active, for each solution $u$ of (6.11)

$$
\frac{u(x)}{\phi(x)} \rightarrow A, \quad \frac{A \phi(x)-u(x)}{\phi_{*}(x)} \rightarrow B
$$

as $x \rightarrow \beta_{2}$

where $A$ and $B$ are constants. To arbitrarily prescribed $A, B$ there exists exactly one $u$ satisfying (6.12). In particular $u_{* 2} \phi^{-1} \rightarrow 0$.

(The parts of Theorems 6.2-6.3 relating to nonactive boundaries can be translated in like manner.)

It follows in particular that when $\beta_{2}$ is active

$$
\int_{\beta}^{\beta 2} u^{2} d m<\infty
$$

for each solution $u$ of (6.11). In other words, if $\beta_{2}$ is active for $\mathfrak{A}_{\psi}$, it is active also for $\lambda-\mathfrak{A}_{\psi}$. The corresponding statement for classical differential operators was formulated by $\mathrm{H}$. Weyl [7].

\section{Green functions}

As before let $\psi>0$ be continuous in the open interval $I:\left(\beta_{1}, \beta_{2}\right)$. We recall from Lemma 5.3 that for any two solutions of $\lambda u-\mathfrak{A}_{\psi} u=0$ we have

$$
u_{2}^{2}\left(u_{1} / u_{2}\right)^{\prime}=\text { const. }
$$

and this constant is zero only if $u_{1}$ and $u_{2}$ are dependent.

Definition 7.1. For fixed $\lambda$ let $u_{1}$ and $u_{2}$ be two independent solutions of $\lambda u-\mathfrak{A}_{\psi} u=0$ normed by the condition

$$
u_{2}^{2}\left(u_{1} / u_{2}\right)^{\prime}=1 .
$$


The Green function $G_{\lambda}$ for $\left(\mathfrak{A}_{\psi}, I\right)$ corresponding to the ordered pair $u_{1}, u_{2}$ is the function of two variables defined by

$$
G_{\lambda}(x, y)=\left\{\begin{array}{lll}
u_{1}(x) u_{2}(y) & \text { for } & \beta_{1}<x<y<\beta_{2} \\
u_{2}(x) u_{1}(y) & \text { for } & \beta_{1}<y<x<\beta_{2} .
\end{array}\right.
$$

We denote by $\mathbb{W}_{\lambda}$ the transformation which takes a function defined in $I$ into the function $F=\mathbb{S}_{\lambda} f$ defined for $x \in I$ by

$$
F(x)=\int_{\beta_{1}}^{\beta_{2}} G_{\lambda}(x, y) f(y) d m(y),
$$

the domain of $\mathbb{S}_{\lambda}$ consisting of those $f$ for which the integral exists in the sense of absolute convergence.

The Green function $G_{\lambda}$, and the transformation ${ }_{B_{\lambda}}$, will be called minimal if $u_{i}$ is minimal at the boundary $\beta_{i}, i=1,2$. (Cf. Theorem 6.1.)

The basic mapping described in Section 4 takes the operator $\mathfrak{A}_{\psi}$ into the operator $\mathfrak{2}^{*}=D_{\mu} D_{\xi}$ defined in an interval $I^{*}:\left(\beta_{1}^{*}, \beta_{2}^{*}\right)$. The variable $x$ becomes a function of $\xi$, and the solutions $u_{i}$ are mapped into solutions $u_{i}^{*}$ of

$$
\lambda u_{i}^{*}-D_{\mu} D_{\xi} u_{i}^{*}=0
$$

where $u_{i}^{*}(\xi)=u_{i}(x) / \psi_{i}(x)$.

The norming condition (7.1) can be rewritten as

$$
\psi^{2}\left\{\left(\frac{u_{1}}{\psi}\right)^{\prime} \frac{u_{2}}{\psi}-\frac{u_{1}}{\psi}\left(\frac{u_{2}}{\psi}\right)^{\prime}\right\}=1
$$

or

$$
u_{2}^{*} \cdot D_{\xi} u_{1}^{*}-u_{1}^{*} \cdot D_{\xi} u_{2}^{*}=1 .
$$

Thus the ordered pair $u_{1}^{*}, u_{2}^{*}$ satisfies the norming condition (7.1) relative to the operator $\mathfrak{A}^{*}$, and we can form the Green function $G_{\lambda}^{*}$ corresponding to ( $\left.\mathfrak{H}^{*}, I^{*}\right)$, namely

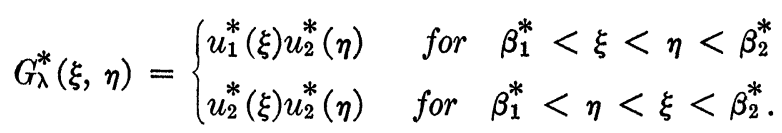

It induces the transformation $\mathcal{S}_{\lambda}^{*}$ taking $f^{*}$ into the function $F^{*}$ defined by

$$
F^{*}(\xi)=\int_{\beta_{1}^{*}}^{\beta_{2}^{*}} G_{\lambda}^{*}(\xi, \eta) f^{*}(\eta) d \mu(\eta) .
$$

A simple inspection shows now that the function $F^{*}$ defined in $I^{*}$ by $(7.8)$ is identical with the image $F / \psi$ of $F=\left(B_{\lambda} f\right.$ under the mapping (4.4). We have thus the simple but useful

Theorem 7.1. The mapping of Section 4 taking the operator $\mathfrak{A}_{\psi}$ into the related operator $\mathfrak{A}^{*}=D_{\mu} D_{\xi}$ induces an isomorphism between the corresponding 
transformations $\mathfrak{S}_{\lambda}$ and $\mathfrak{S}_{\lambda}^{*}$. If $\mathfrak{S}_{\lambda}$ is minimal for $\mathfrak{A}_{\psi}$, then $\mathfrak{S}_{\lambda}^{*}$ is minimal for $\mathfrak{Y} *$.

Consequently, we may restrict further consideration to operators of the simple form $\mathfrak{A}=D_{m} D_{x}$ and reformulate all results for the general $\mathfrak{A}_{\psi}$. The advantage of considering only the operator $\mathfrak{A}$ is merely formal: the formulas become more pleasing to the eye, and the argument is more intuitive due to the fact that the positive solutions of $\lambda u-\mathfrak{A} u=0$ are convex.

Theorem 7.2. Let $G_{\lambda}$ be a Green function for $\mathfrak{A}=D_{m} D_{x}$ and $I:\left(\beta_{1}, \beta_{2}\right)$, formed by means of two solutions $u_{i}$ such that $u_{1}$ is increasing and $u_{2}$ decreasing. Then

(i) Each bounded continuous $f$ is mapped into a bounded continuous $F$ such that

$$
\lambda F-D_{m} F^{\prime}=f
$$

Furthermore,

$$
0 \leqq f \leqq 1 \text { implies } 0 \leqq \lambda F \leqq 1
$$

(ii) If $f$ is square integrable with respect to $m$, then $F$ is bounded and absolutely continuous with respect to $m$; the equation (7.9) holds m-almost everywhere, and

Proof. We have

$$
\lambda^{2} \int_{\beta_{1}}^{\beta_{2}} F^{2} d m \leqq \int_{\beta_{1}}^{\beta_{2}} f^{2} d m
$$

$$
F(x)=u_{1}(x) \int_{x+}^{\beta_{2}} f(y) u_{2}(y) d m(y)+u_{2}(x) \int_{\beta_{1}}^{x+} f(y) u_{1}(y) d m(y) .
$$

The solutions $u_{i}$ being monotonic, it follows directly from Lemma 6.1 that the integrals converge absolutely for each bounded $f$. For the special choice $f=1$ we get, remembering that $u_{1}^{+} u_{2}-u_{1} u_{2}^{+}=1$ and the monotonicity of $u_{i}$,

$$
\begin{aligned}
\lambda F(x) & =u_{1}(x) \int_{x+}^{\beta_{2}} d u_{2}^{\prime}(y)+u_{2}(x) \int_{\beta_{1}}^{x+} d u_{1}^{\prime}(y) \\
& =1+u_{1}(x) u_{2}^{-}\left(\beta_{2}\right)-u_{2}(x) u_{1}^{+}\left(\beta_{1}\right) \leqq 1 .
\end{aligned}
$$

This proves (7.10).

If $f$ is continuous and $m$ has a continuous derivative with respect to $x$, then (7.12) may be differentiated formally to obtain

$$
F^{+}(x)=u_{1}^{+}(x) \int_{x+}^{\beta_{2}} f(y) u_{2}(y) d m(y)+u_{2}^{+}(x) \int_{\beta_{1}}^{x+} f(y) u_{1}(y) d m(y)
$$

To verify the general validity of (7.14) denote the right hand member by $\Phi(x)$. A simple integration by parts shows that

$$
\int_{\alpha}^{\beta} \Phi(x) d x=F(\beta)-F(\alpha)
$$


showing that $F^{+}$equals almost everywhere a derivative of $F$. The right continuity of $\Phi$ implies that $\Phi=F^{+}$everywhere. Now each term in (7.14) is continuously differentiable with respect to $m$; remembering that $D_{m} u_{i}^{+}=$ $\lambda u_{i}$ and $u_{1}^{+} u_{2}-u_{2}^{+} u_{1}=1$, we see that differentiation of (7.14) leads to (7.9). This proves the first part of the theorem.

Suppose that $f$ is square integrable. Lemma 6.1 implies that $u_{2}$ is square integrable over each interval $\left(x, \beta_{2}\right)$, and therefore the first integral in (7.12) converges absolutely. A similar argument applies to the second integral, and a trivial modification of the above reasoning shows the validity of (7.13) and the assertion relative to (7.9). It remains to prove (7.11). Now by Schwarz' inequality and (7.10)

$$
\begin{aligned}
\lambda^{2} F^{2}(x) & \leqq \lambda^{2} \int_{\beta_{1}}^{\beta_{2}} G_{\lambda}(x, y) f^{2}(y) d m(y) \cdot \int_{\beta_{1}}^{\beta_{2}} G_{\lambda}(x, y) d m(y) \\
& \leqq \lambda \int_{\beta_{1}}^{\beta_{2}} G_{\lambda}(x, y) f^{2}(y) d m(y) .
\end{aligned}
$$

Integrate this inequality with respect to $m$ using Fubini's theorem. Since $G_{\lambda}$ is symmetric, a renewed application of (7.10) leads to (7.11). The theorem is proved.

\section{The minimal transformation}

In this section $\lambda$ is an arbitrary positive number.

Theorem 8.1. Let $\mathbf{H}_{m}$ be the Hilbert space defined by (1.5). Let $G_{\lambda}$ be the minimal Green function for the operator $\mathfrak{A}=D_{m} D_{x}$ and the interval

$$
I:\left(\beta_{1}<x<\beta_{2}\right) \text {. }
$$

The corresponding transformation ${ }^{14}{ }^{1}{ }_{\lambda}$ of $(7.3)$ is a symmetric, self-adjoint transformation with norm $\left\|\mathrm{S}_{\lambda}\right\|_{m} \leqq \lambda^{-1}$. Its domain is the entire Hilbert space $\mathbf{H}_{m}$, and its range $R$ is defined as follows:

(i) If neither $\beta_{1}$ nor $\beta_{2}$ is active, the range $R$ coincides with the set $\Delta$ of all functions $F$ such that $\mathfrak{A} F$ exists m-almost everywhere and both $F$ and $\mathfrak{A} F$ are square integrable $(m)$.

(ii) If $\beta_{i}$ is active, the range $R$ is the subset of $\Delta$ consisting of those functions for which

$$
\begin{array}{lll}
F(x) \rightarrow 0 & \text { as } x \rightarrow \beta_{i} & \text { if }\left|\beta_{i}\right|<\infty \\
F(x)=o(|x|) & \text { as } x \rightarrow \beta_{i} & \text { if }\left|\beta_{i}\right|=\infty .
\end{array}
$$

In other words, for arbitrary $f \in \mathbf{H}_{m}$ there exists exactly one solution $F \in R$ of $\lambda F-\mathfrak{A} F=f$. The restriction of $\mathfrak{A}$ to $R$ is a self-adjoint operator.

Note. It is clear from Theorem 7.1 that the present theorem applies

${ }^{14}$ Strictly speaking we are dealing with the restriction of ${ }^{S_{\lambda}}$ to $\mathbf{H}_{m}$ (and, similarly, in the next theorem with the restriction to $\mathbf{C}$ ). It seems foolish, however, to introduce a new letter in each case. 
equally to arbitrary operators $\mathfrak{A}_{\psi}$, the general formulation of the boundary condition (8.1) being: if $\beta_{i}$ is active, then

$$
F(x)=o(\phi(x)) \text { as } x \rightarrow \beta_{i}
$$

where $\phi$ is an arbitrary solution of $\mathfrak{P}_{\psi} \phi=0$ which is positive but not minimal at $\beta_{i}$. The range $R$ is independent of $\lambda$, and thus $\mathfrak{S}_{\lambda}$ is the resolvent of $\mathfrak{A}$ if no boundary is active, and the resolvent of the restriction $\mathfrak{V} / R$ otherwise. The range $R$ is obviously dense in the Hilbert space $\mathbf{H}_{m}$.

Proof. By Theorem 7.2 the transformation $\mathscr{S}_{\lambda}$ is positive, symmetric, and has $\mathbf{H}_{m}$ for domain. It is therefore self-adjoint. The inequality (7.11) implies $\left\|S_{\lambda}\right\|_{m} \leqq \lambda^{-1}$. We next show that the range of $\mathscr{S}_{\lambda}$ is contained in $R$. This statement is tautological if neither boundary is active. Suppose, then, that $\beta_{2}$ is active. Formula (7.12) applies with $u_{i}$ standing for the solution $u_{* i}$ which is minimal at $\beta_{i}$. By Theorems 6.2 and 6.3 the the solution $u_{1}$ is square integrable over $\left(\beta_{1}, \beta_{2}\right)$, and therefore the first integral in (7.12) approaches 0 as $x \rightarrow \beta_{2}$ while the second remains bounded. If $\beta_{2}<\infty$, then $u_{1}$ is bounded and $u_{2} \rightarrow 0$ as $x \rightarrow \beta_{2}$. If $\beta_{2}=\infty$, then $u_{1}=O(x)$ and $u_{2}=O(1)$. In the first case $F(x) \rightarrow 0$, in the second $F(x)=O(1)$, as asserted. It remains to prove that, conversely, $R$ is contained in the range of $\left(S_{\lambda}\right.$. Choose an arbitrary $F_{0} \epsilon R$, and set $f=\lambda F_{0}-\mathfrak{A} F_{0}$. Then $f$ is square integrable, $F_{0} \epsilon R$, and $F=\oiint_{\lambda} f \epsilon R$. It follows that $u=F_{0}-F$ is a solution of $\lambda u-\mathfrak{A} u=0$ and $u \epsilon R$. However, by Theorems 6.2 and 6.3, $u=0$ is the only solution with these properties, and hence $F=F_{0}$ as asserted.

Theorem 8.2. Let $\mathbf{C}$ be the Banach space of functions continuous in the closed interval $\left[\beta_{1}, \beta_{2}\right]$ with the norm (1.17). Let $G_{\lambda}$ be the minimal Green function for $\mathfrak{A}=D_{m} D_{x}$ and $I:\left(\beta_{1}, \beta_{2}\right)$.

The transformation $\mathbb{S}_{\lambda}$ is a positive linear transformation from $\mathbf{C}$ to $\mathbf{C}$ with norm $\left\|\mathrm{G}_{\lambda}\right\| \leqq \lambda^{-1}$. Its domain is $\mathbf{C}$, and the range $R$ is defined as follows: Let $\Delta$ be the set of all functions $F \in \mathbf{C}$ such that $\mathfrak{A} F \in \mathbf{C}$.

(i) $R=\Delta$ if, and only if, the boundaries are at infinity or, in case of $\left|\beta_{i}\right|<\infty$, this $\beta_{i}$ is neither active nor semiactive. ${ }^{15}$

(ii) If $\left|\beta_{i}\right|<\infty$ and $\beta_{i}$ is either active or semiactive, then

$$
F(x) \rightarrow 0 \text { as } x \rightarrow \beta_{i}
$$

for each $F \in R$, and $R$ consists of all $F \in \Delta$ satisfying (8.3).

In other words, for each $f \in \mathbf{C}$ there exists exactly one $F \in R$ satisfying the equation $\lambda F-\mathfrak{U} F=f$. The operator $\mathfrak{S}_{\lambda}$ is the resolvent of $\mathfrak{A}$ or of the restriction $\mathfrak{U} / R$.

Proof. Referring again to Theorem 7.2, we first prove that $F \in \mathbf{C}$, that is, that $F(x)$ approaches limits as $x \rightarrow \beta_{i}$. Consider two special cases. (i) Suppose that $f$ vanishes identically in some neighborhood of $\beta_{2}$. In this neigh-

\footnotetext{
${ }^{15}$ In the nomenclature of footnote 12 it is required that neither boundary be regular or exit.
} 
borhood $F$ reduces to a constant multiple of $u_{2}$, and therefore a finite limit $F\left(\beta_{2}\right)$ exists. In particular, $F\left(\beta_{2}\right)=0$ when $\beta_{2}<\infty$. (ii) Let $f=1$. By (7.13) in this case $\lambda F=1-p_{1} u_{1}-p_{2} u_{2}$ (where $p_{1}=0$ if $u_{1}$ is unbounded at $\left.\beta_{2}\right)$. Again it is clear that $F$ approaches a limit $F^{\prime}\left(\beta_{2}\right)$ and $0 \leqq F\left(\beta_{2}\right) \leqq \lambda^{-1}$. We are now in a position to consider an arbitrary $f \in \mathbf{C}$. It can be split into a sum $f=f\left(\beta_{2}\right) 1+f_{1}+f_{2}$ of three functions, of which the first is constant, the second vanishes identically in some neighborhood of $\beta_{2}$, and the third satisfies the inequality $\left|f_{2}\right|<\varepsilon$. The transform of $f\left(\beta_{2}\right) 1+f_{1}$ has been shown to approach a limit as $x \rightarrow \beta_{2}$. By (7.10) the transform $F_{2}=B_{\lambda} f_{2}$ satisfies the inequality $\lambda\left\|F_{2}\right\|<\varepsilon$. Accordingly, the oscillation of $F=\mathcal{B}_{\lambda} f$ near $\beta_{2}$ is smaller than $\varepsilon \lambda^{-1}$, and it follows that the limit $F\left(\beta_{2}\right)$ exists. $^{16}$

From (7.10) we see that $\left\|G_{\lambda}\right\| \leqq \lambda^{-1}$. To show that each $F=B_{\lambda} f$ satisfies (8.3) suppose that $\beta_{2}<\infty$ and that $\beta_{2}$ is semiactive. Then $u_{2} \downarrow 0$, while $u_{1}$ remains bounded at $\beta_{2}$. It follows that the first term on the right in (7.12) approaches 0 as $x \rightarrow \beta_{2}$, and it suffices to prove that $u_{2}(x) m(x) \rightarrow 0$. Now for $x>\beta$

$$
u_{2}(x) m(x)<u_{2}(x) m(\beta)+\int_{\beta}^{x} u_{2}(y) d m(y)<u_{2}(x) m(\beta)+\varepsilon
$$

provided $\beta$ is chosen close enough to $\beta_{2}$. It follows that lim sup $u_{2}(x) m(x)<$ $\varepsilon$ or $u_{2} m \rightarrow 0$ as asserted. When $\beta_{2}$ is finite and active, the fact that $F \rightarrow 0$ is trivial since $m$ remains bounded. We have thus proved that the range of (S) is contained in $R$. The last step of the proof of Theorem 8.1 applies here without change to show that $R$ is contained in the range of $G_{\lambda}$, and this completes the proof.

The contrast between the last two theorems is striking. An infinite active boundary gives rise to nonuniqueness (i.e. a boundary condition) in the Hilbert space $\mathbf{H}_{m}$, but not in $\mathbf{C}$; a finite semiactive boundary gives a boundary condition in $\mathbf{C}$ but not in $\mathbf{H}_{m}$. Actually a finite semiactive boundary may be transformed into an infinite semiactive boundary (Section 4), and it is clear therefore that in the latter case the absence of a boundary condition is imposed only by the extraneous norm condition.

To be specific, the last theorem asserts that

$$
0 \leqq \lambda\left(S_{\lambda} 1 \leqq 1\right.
$$

and the second equality sign holds only in the case of uniqueness (i). Exactly the same argument can be applied not only to the function 1, but to any positive solution $\phi$ of $\mathfrak{2} \phi=0$. For example, if the basic interval $I$ is $(0, \infty)$ we get

$$
0 \leqq \lambda\left(\uplus_{\lambda} x \leqq x,\right.
$$

${ }^{16}$ For an application in Section 9 we note that we have proved $\lambda F\left(\beta_{2}\right) \leqq f\left(\beta_{2}\right)$ if $f>0$ and $\beta_{2}<\infty$. [Incidentally, this is not true if $\beta_{2}=\infty$ and $\infty$ is semiactive.] 
with the second equality sign holding if infinity is neither an active nor a semiactive boundary, in which case $\lambda\left(S_{\lambda} x=o(x)\right.$ as $x \rightarrow \infty$. Theorem 8.2 is contained in the following which does not refer to any particular norm and shows how $\$_{\lambda}$ acts on unbounded functions.

Theorem 8.3. Suppose $-\infty \leqq \beta_{1}<0<\beta_{2} \leqq \infty$. Let $\mathbf{L}$ be the set of all functions $f$ which are continuous in the open interval $I:\left(\beta_{1}, \beta_{2}\right)$ and for which $f(x) x^{-1}$ tends to a finite limit as $x \rightarrow \beta_{i}$. Let $\Delta$ be the subset of those $F \in \mathbf{L}$ for which $\mathfrak{A} F \in \mathbf{L}$ (where $\mathfrak{A}=D_{m} D_{x}$ ). Let $\mathfrak{S}_{\lambda}$ be the transformation (7.3) associated with the minimal Green function for ( $\mathfrak{A}, I$ ).

Then $\mathbb{S}_{\lambda}$ is a closed linear transformation from $\mathbf{L}$ to $\mathbf{L}$. Its domain is $\mathbf{L}$, and its range $R$ coincides with $\Delta$ if, and only if, neither boundary is active or semiactive. If $\beta_{i}$ is active or semiactive, then $R$ coincides with the subset of those $F \in \Delta$ for which

$$
F(x) x^{-1} \rightarrow 0
$$$$
x \rightarrow \beta_{i} \text {. }
$$

The proof is a mere repetition of the preceding one; the assertion follows also from Theorem 8.2 by the standard mapping procedure introduced at the end of Section 4.

Corollary to Theorem 8.2. Let $\mathbf{C}_{\psi}$ be the Banach space of functions continuous in I metrized by the norm (1.18). The minimal Green function for $\mathfrak{A}_{\psi}$ induces a bounded transformation $\mathfrak{S}_{\lambda}$ from $\mathbf{C}_{\psi}$ to $\mathbf{C}_{\psi}$ with norm $\lambda^{-1}$.

It is clear that in this statement $\psi$ may be replaced by an arbitrary positive solution of $\mathfrak{A}_{\psi} \phi=0$, and that the boundary conditions are inferred from (8.3).

Note on the term "minimal". It is readily seen from Theorems 8.1 and 8.2 that for any positive bounded transformation $\bar{G}_{\lambda}$ from $\mathbf{C}$ to $\mathbf{C}$ (or from $\mathbf{H}_{m}$ to $\mathbf{H}_{m}$ ) which transforms each $f$ into a solution $F$ of $\lambda F-\mathfrak{A} F=f$ one has $\bar{G}_{\lambda} f \geqq$ ( $_{\lambda} f$ for each $f \geqq 0$. This observation will be amplified in Sections 10 and 12 .

\section{The role of convexity}

We have just seen that for the most general operator $\mathfrak{A}_{\psi}$, defined by an arbitrary continuous $\psi>0$, there exists a minimal Green function for each $\lambda>0$. It leads to an inversion $\mathfrak{B}_{\lambda}$ of $\lambda-\mathfrak{A}_{\psi}$, and $\mathfrak{S}_{\lambda}$ is a positive operator such that $\lambda \mathscr{S}_{\lambda} \psi \leqq \psi$. This means that with the norm (1.18) $\lambda\left(S_{\lambda}\right)_{\text {becomes }}$ norm nondecreasing. Unfortunately we are not always free to accept this norm, and very often we are tied to the traditional Banach norm (1.17). The question then arises (in particular in probability theory and heat conduction) whether the operator $\lambda\left(\$_{\lambda}\right.$ corresponding to $\mathfrak{P}_{\psi}$ is norm preserving or decreasing ("a transition operator") when the familiar norm (1.17) is used. In other words, we ask: what conditions must $\psi$ satisfy in order that

$$
\lambda\left(S_{\lambda} 1 \leqq 1\right. \text {. }
$$

The answer is found in 
Theorem 9.1. Let ${\left(\$_{\lambda}\right.}_{\lambda}$ be the transformation (7.3) associated with the minimal Green function for $\left(\mathfrak{A}_{\psi}, I\right)$ where $\psi>0$ in I. In order that (9.1) hold for all $\lambda>\lambda_{0}$, it is necessary and sufficient that $\psi$ be convex downward (the graph of $\psi$ lying below the chords).

In this case each positive solution $\phi$ of $\mathfrak{A}_{\psi} \phi=0$ is convex downward, and (9.1) holds for each $\lambda>0$.

Proof. In the special case $\psi=1$ the operator $\mathfrak{A}_{\psi}$ reduces to $\mathfrak{U}=D_{m} D_{x}$ : the relation (9.1) is then contained in (7.10) and all solutions of $\mathfrak{A}_{\psi} \phi=0$ are linear functions, hence convex. The assertion is therefore true in the special case $\psi=$ const., and we shall from now on assume that $\psi$ is not constant.

It is convenient to break up the remainder of the proof into minor lemmas.

LEMMA 9.1. If $\psi$ is convex downward, so is each positive solution $\phi$ of $\mathfrak{A}_{\psi} \phi=0$.

Proof. Since $\psi$ is convex downward and not constant, it either decreases near $\beta_{1}$ or increases near $\beta_{2}$. Assume the latter. Being convex, $\psi$ increases at least linearly, and hence the integral over $\psi^{-2}(x) d x$ converges at $\beta_{2}$. It follows that

$$
\phi(x)=\psi(x) \int_{x}^{\beta_{2}} \psi^{-2}(s) d s
$$

defines that solution of $\mathfrak{A}_{\psi} \phi=0$ which is minimal at $\beta_{2}$. We prove that this $\phi$ is convex downward.

In view of the assumed convexity, $\psi$ possesses a monotonically increasing right derivative $\psi^{+}$. From (9.2) we see that also the right derivative

$$
\phi^{+}(x)=\psi^{+}(x) \int_{x}^{\beta_{2}} \psi^{-2}(s) d s-\psi^{-1}(x)
$$

exists for each $x \in I$. For $x<x+h<\beta_{2}$ we have

$$
\begin{aligned}
& \phi^{+}(x+h)-\phi^{+}(x)=\left\{\psi^{+}(x+h)-\psi^{+}(x)\right\} \int_{x+h}^{\beta_{2}} \psi^{-2}(s) d s \\
& \quad-\psi^{+}(x) \int_{x}^{x+h} \psi^{-2}(s) d s-\psi^{-1}(x+h)+\psi^{-1}(x) \\
& \geqq-\int_{x}^{x+h} \psi^{+}(s) \cdot \psi^{-2}(s) d s-\psi^{-1}(x+h)+\psi^{-1}(x)=0 .
\end{aligned}
$$

Hence $\phi^{+}$increases and $\phi$ is convex. If $\psi$ is not minimal at $\beta_{1}$, then the same argument applies at $\beta_{1}$, and we see that the two minimal solutions are linearly independent and convex. Every positive solution of $\mathfrak{A}_{\psi} \phi=0$ being a positive linear combination of the minimal solutions, the lemma is proved.

Lemma 9.2. Let $F_{\lambda}=\left(\$_{\lambda} f\right.$ be the transform defined by (7.3) when $G_{\lambda}$ is the minimal Green function for $\mathfrak{U}=D_{m} D_{x}$. If $f$ is continuous in $I$ and $f(x) \rightarrow 0$ as $x \rightarrow \beta_{i}(i=1,2)$, then $\lambda F_{\lambda} \rightarrow f$ as $\lambda \rightarrow \infty$ uniformly in $I$. 
Proof. (This lemma is known and proved for completeness only.) Suppose first that $f \in R$, where $R$ is the range described in Theorem 8.2. Then $\mathfrak{A} f$ exists, and we conclude from

$$
\begin{gathered}
\lambda F_{\lambda}-\mathfrak{T} F_{\lambda}=f \\
\lambda \mathfrak{U} F_{\lambda}-\mathfrak{U}^{2} F_{\lambda}=\mathfrak{A} f,
\end{gathered}
$$

and that $\mathfrak{U} F_{\lambda} \in R$. It follows that $\mathfrak{U} F_{\lambda}=\mathfrak{G}_{\lambda}(\mathfrak{U} f)$, and Theorem 8.2 asserts that $\lambda\left\|\mathfrak{A} F_{\lambda}\right\| \leqq\|\mathfrak{U} f\|$. Thus from (9.5)

$$
\left\|\lambda F_{\lambda}-f\right\| \leqq \lambda^{-1}\|\mathfrak{Y} f\| \rightarrow 0,
$$

$\lambda \rightarrow \infty$.

The assertion is therefore proved for $f \in R$. However, each $f$ which vanishes at the boundaries is in the closure of $R$, and a routine application of the triangle inequality shows that $\left\|\lambda F_{\lambda}-f\right\| \rightarrow 0$ for each $f$ in this closure.

Lemma 9.3. Adhering to the notations of the preceding lemma, suppose that there exists a function $f>0$ such that

$$
\lambda F_{\lambda} \leqq f
$$

for each $\lambda>\lambda_{0}$. Then $f>0$ is convex upward.

Conversely, suppose that $f$ is convex upward and that the interval $I$ is finite $\left(-\infty<\beta_{1}<\beta_{2}<\infty\right)$. Then (9.8) holds for all $\lambda>0$.

Proof. A comparison of (9.8) and (9.5) shows that (9.8) is equivalent to $\mathfrak{A} F_{\lambda} \leqq 0$. In this case $D_{x} F_{\lambda}$ decreases, and $F_{\lambda}$ is convex upward. Let $I^{(0)}$ be a closed subinterval of $I$, and construct a function $f^{(0)}$ such that $f^{(0)}(x)=$ $f(x)$ for $x \epsilon I^{(0)}$ and $0 \leqq f^{(0)}(x) \leqq f(x)$ for $x \epsilon I$ and $f^{(0)}(x) \rightarrow 0$ as $x \rightarrow \beta_{i}$. Put $F_{\lambda}^{(0)}=\$_{\lambda} f^{(0)}$. By Lemma 9.2

$$
\lambda F_{\lambda}(x) \geqq \lambda F_{\lambda}^{(0)}(x) \rightarrow f^{(0)}(x)=f(x),
$$$$
x \in I^{(0)} \text {. }
$$

It follows that $\lambda F_{\lambda}(x) \rightarrow f(x)$ for each $x \in I$ and hence $f$ is convex upward. This proves the first part of the lemma.

Conversely, suppose that $f$ is convex upward and $\left|\beta_{i}\right|<\infty$. We recall the footnote 15 to Theorem 8.2 according to which

$$
\lambda F_{\lambda}\left(\beta_{i}\right) \leqq f\left(\beta_{i}\right),
$$$$
i=1,2 .
$$

Suppose now that (9.8) is false, and let $I^{\prime}=\left(x_{1}, x_{2}\right)$ be a maximal subinterval of $I$ such that $\lambda F_{\lambda}(x)>f(x)$ for $x \epsilon I^{\prime}$. In view of (9.10) the endpoints of $I^{\prime}$ must be interior points, i.e.

$$
\lambda F_{\lambda}\left(x_{i}\right)-f\left(x_{i}\right)=0,
$$$$
i=1,2 .
$$

But inside $I^{\prime}$ we have $\mathfrak{A} F_{\lambda}>0$. Hence $F_{\lambda}$ is convex downward, and the same is true of the difference $\lambda F_{\lambda}-f$. Remembering (9.11) we conclude that $\lambda F_{\lambda}(x)<f(x)$ for $x \in I^{\prime}$, which contradicts the definition of $I^{\prime}$. The lemma is proved. 
Completion of the proof. Suppose that (9.1) holds for all $\lambda>\lambda_{0}$, and put $\$_{\lambda} 1=H_{\lambda}$. Using Theorem 7.1 we wish to reformulate (9.1) in terms of the related operator $\mathfrak{2}^{*}=D_{\mu} D_{\xi}$. The variable $x$ becomes a function of $\xi$, and $H_{\lambda}$ and 1 are mapped, respectively, into the functions defined by $F_{\lambda}(\xi)=$ $H_{\lambda}(x) \psi^{-1}(x)$ and $f(\xi)=\psi^{-1}(x)$. Here $F_{\lambda}=\mathcal{S}_{\lambda}^{*} f$, where $\mathcal{S}_{\lambda}^{*}$ corresponds to the minimal Green function for $\mathfrak{A}^{*}$. Now (9.1) states that $\lambda F_{\lambda} \leqq f$ for $\lambda>\lambda_{0}$, and the preceding lemma shows that $f$ is convex upward, i.e. that $D_{\xi}^{+} f$ is decreasing. But $D_{\xi}^{+} f=\psi^{2} D_{x}^{+}\left(\psi^{-1}\right)=-D_{x}^{+} \psi$, and therefore $\psi$ is convex downward.

Conversely, suppose that $\psi$ is convex downward. By Lemma 9.1 all positive solutions of $\mathfrak{A}_{\psi} \phi=0$ are convex downward, and since $\mathfrak{A}_{\psi}=\mathfrak{A}_{\phi}$, there is no loss of generality in assuming that $\psi$ is not minimal either at $\beta_{1}$ or $\beta_{2}$. The transformation (4.1) then maps the interval $I=\left(\beta_{1}, \beta_{2}\right)$ on a finite interval $I^{*}=\left(\beta_{1}^{*}, \beta_{2}^{*}\right)$, and $f$ becomes a function of $\xi$ which is convex upward. The last lemma then asserts that $\lambda F_{\lambda} \leqq f$ for all $\lambda>0$, and the theorem is proved.

\section{Self-adjoint operators}

We consider the Hilbert space $\mathbf{H}_{m}$ defined by (1.5) and propose to investigate the most general closed symmetric linear operator ${ }^{3} \lambda$ which, for a fixed $\lambda>0$, associates with each $f \in \mathrm{H}_{m}$ an element $F \epsilon \mathrm{H}_{m}$ such that
(10.1) G
$\lambda F-\mathfrak{A} F=f$,
$\mathfrak{A}=D_{m} D_{x}$

for almost all $x \epsilon I=\left(\beta_{1}, \beta_{2}\right)$. Since the domain of $\left.{ }^{\prime}\right\rangle_{\lambda}$ is the entire space, $B_{\lambda}$ is a bounded self-adjoint operator.

Let $\oint_{\lambda}^{\min }$ be the minimal Green function for $(\mathfrak{A}, I)$, and $\mathfrak{S}_{\lambda}^{\min }$ the induced transformation. Both $F=\mathbb{S}_{\lambda} f$ and $F^{\text {min }}=\mathscr{S}_{\lambda}^{\text {min }} f$ satisfy (10.1), and therefore $w=F-F^{\mathrm{min}}$ must be a solution of

$$
\lambda w-\mathfrak{A} w=0 .
$$

Let $w_{1}$ and $w_{2}$ be two independent solutions of (10.2). It is then clear that

$$
\mathfrak{S}_{\lambda} f=\mathfrak{S}_{\lambda}^{\min } f+w_{1} \cdot \Phi_{f}^{(1)}+w_{2} \cdot \Phi_{f}^{(2)},
$$

where $\Phi_{f}^{(2)}$ stands for a bounded linear functional, i.e. an element of $\mathbf{H}_{m}$. It follows that $\left.{ }^{3}\right\rangle_{\lambda}$ is a kernel transformation with $F=\mathcal{G}_{\lambda} f$ being defined by

$$
F(x)=\int_{\beta_{1}}^{\beta_{2}} G_{\lambda}(x, y) f(y) d m(y),
$$

where

$$
G_{\lambda}(x, y)=G_{\lambda}^{\min }(x, y)+w_{1}(x) \Phi^{(1)}(y)+w_{2}(x) \Phi^{(2)}(y) .
$$

The further condition that $\oiint_{\lambda}$ be symmetric reduces (10.5) to the form

$$
G_{\lambda}(x, y)=G_{\lambda}^{\min }(x, y)+\sum_{\imath, j=1}^{2} p_{i j}(\lambda) w_{\imath}(x) w_{\jmath}(y),
$$

where the $p_{i \jmath}(\lambda)$ are constants, $p_{12}(\lambda)=p_{21}(\lambda)$. 
If $w_{1}$ and $w_{2}$ (i.e. if all solutions of (10.2)) are square integrable $(m)$, then the symmetric matrix $P(\lambda)=\left(p_{i j}(\lambda)\right)$ may be chosen arbitrarily, and (10.6) defines a transformation of the required form. Such is the case when both boundaries are active. If only $\beta_{2}$ is acțive, three coefficients $p_{i j}(\lambda)$ must vanish; if neither endpoint is active, the minimal $\left(s_{\lambda}^{\min }\right.$ is the only transformation of the desired type.

When for each $\lambda>0$ the coefficients $p_{i j}(\lambda)$. are chosen in an arbitrary manner, no natural connection between the corresponding $\mathcal{S}_{\lambda}$ must be expected. In particular, even though $(\lambda-\mathfrak{U}) \mathfrak{S}_{\lambda}$ reduces to the identity, $\mathfrak{S}_{\lambda}$ will not represent the resolvent of any fixed operator. Only if the range $R$ of $B_{\lambda}$ is independent of $\lambda$ does $\left(S_{\lambda}\right.$ represent the resolvent ${ }^{17}$ of an operator, namely $\mathfrak{S S}_{\lambda}=\{\lambda-\mathfrak{U} / R\}^{-1}$ where $\mathfrak{A} / R$ is the restriction of $\mathfrak{U}$ to $R$. This $\mathfrak{A} / R$ as well as $\$_{\lambda}$ is then self-adjoint.

We are thus confronted with the following questions:

(1) Given a self-adjoint $\$_{\lambda}$, determine its range $R$.

(2) Describe the most general possible range $R$.

(3) Given $R$, determine the coefficients $p_{i j}(\lambda)$ in such a way that $\left(\$_{\lambda}\right.$ will have the prescribed range $R$.

(4) Find the conditions under which the transformation $\left(S_{\lambda}\right.$ will be positive, or norm-decreasing, etc.

The case of one active boundary is simpler than the case of two active boundaries, and can be derived from it in trivial fashion by equating certain coefficients to zero. We shall therefore suppose that both $\beta_{1}$ and $\beta_{2}$ are active. First we take up the case where $\left|\beta_{i}\right|<\infty$. It will be observed that the same calculations apply to infinite intervals (and, indeed, to the general operator $\mathfrak{A}_{\psi}$ ). The only advantage (and only purpose) in a separate treatment of finite intervals is the appearance of the classical boundary conditions under their traditional form, unobscured by a new symbolism.

Theorem 10.1. Let $\beta_{1}$ and $\beta_{2}$ be active boundaries, $\left|\beta_{i}\right|<\infty$. For some fixed $\lambda>0$ let $\mathcal{S S}_{\lambda}$ be a bounded self-adjoint transformation taking each $f \in \mathbf{H}_{m}$ into $\mathbb{S}_{\lambda} f=F \in \mathbf{H}_{m}$ such that (10.1) holds almost everywhere $(m)$. Then:

(a) There exist two boundary conditions which are either of the form ${ }^{18}$

$$
\begin{aligned}
-F^{+}\left(\beta_{1}\right)+\pi_{11} F\left(\beta_{1}\right)+\pi_{12} F\left(\beta_{2}\right) & =0, \\
F^{\prime-}\left(\beta_{2}\right)+\pi_{21} F\left(\beta_{1}\right)+\pi_{22} F\left(\beta_{2}\right) & =0,
\end{aligned}
$$

where

$$
\pi_{12}=\pi_{21}
$$

17 The so-called resolvent equation $\mathscr{S}_{\lambda}-\mathscr{S}_{\nu}=(\nu-\lambda) \mathfrak{S}_{\nu}\left(S_{\lambda}\right.$ is an obvious consequence of (10.1): substracting (10.1) for two values of the parameter we get $\nu\left\{\mathbb{S}_{\nu}-\mathbb{S}_{\lambda}\right\}-$ $A\left\{\mathfrak{G}_{\nu}-\mathfrak{G}_{\lambda}\right\}=(\lambda-\nu) \mathfrak{S}_{\lambda}$. This is equivalent to the resolvent equation if, and only if, the ranges of $\mathbb{S}_{\lambda}$ and $\mathbb{S}_{\nu}$ coincide.

${ }^{18} \mathrm{In}(10.7) \mathrm{it}$ is understood that $F$ is continuous in the closed interval and $F^{-}\left(\beta_{2}\right)=$ $\lim F^{+}(x)=\lim F^{-}(x)$. In (10.8) the case $\pi_{1}=0$ is included with the obvious interpretation that it implies $F\left(\beta_{1}\right)=0$. If $\pi_{1}=\pi_{2}=0$, the boundary conditions (10.8) are $F\left(\beta_{1}\right)=F\left(\beta_{2}\right)=0$, i.e. $\left(\xi_{\lambda}=\mathcal{H}_{\lambda}^{\min }\right.$. 
or of the form

$$
\alpha \pi_{1}^{-1} F\left(\beta_{1}\right)=\alpha \pi_{2}^{-1} F\left(\beta_{2}\right)=\pi_{1} F^{+}\left(\beta_{1}\right)-\pi_{2} F^{-}\left(\beta_{2}\right) .
$$

The range $R$ of $\$_{\lambda}$ consists of those $F \in \mathbf{H}_{m}$ which satisfy the boundary conditions and the trivial requirement $\mathfrak{A} F \in \mathbf{H}_{m}$.

(b) Conversely: to arbitrarily prescribed coefficients $\pi_{i j}$ (or $\left.\pi_{i}, \alpha\right)$ there corresponds a $\lambda_{0}$ such that for each $\lambda>\lambda_{0}$ there exists exactly one transformation $\$_{\lambda}$ of the described sort whose range $R$ is characterized by the prescribed boundary condition.

Note 1. Given the coefficients in the boundary conditions, the explicit form of the coefficients $p_{i j}(\lambda)$ is found in (10.22) for the boundary conditions (10.7), and in (10.23)-(10.25) for the conditions (10.8).

Note 2. If $\pi_{12}=\pi_{21}=0$ in (10.7), the transformation $\$_{\lambda}$ is induced by the Green function $G_{\lambda}$ formed by means of two solutions satisfying one boundary condition each.

Note 3. The condition $\pi_{12}=\pi_{21}$ is imposed merely by the requirement of symmetry. The arguments go through also when $\pi_{12} \neq \pi_{21}$. See Section 12 for more general unsymmetric transformations.

Theorem 10.2. (a) In order that the transformation (5) $_{\lambda}$ of Theorem $10.1 \mathrm{~b}$ be positive for all $\lambda$ sufficiently large, it is necessary and sufficient that $\pi_{12} \leqq 0$ (in the case of the boundary condition (10.7)) or $\pi_{1} \pi_{2} \geqq 0$ (in the case (10.8)).

(b) In order that ${ }^{\left(S_{\lambda}\right.}$ be positive and

$$
\lambda\left(S_{\lambda} 1 \leqq 1\right.
$$

for all $\lambda$ sufficiently large, it is necessary and sufficient that

or

$$
\pi_{12} \leqq 0, \quad \pi_{i 1}+\pi_{i 2} \geqq 0,
$$$$
i=1,2
$$

$$
\pi_{1} \geqq 0, \quad \pi_{2} \geqq 0, \quad \alpha>0,
$$

respectively. In this case $\mathfrak{S}_{\lambda}$ is positive, and (10.9) holds for all $\lambda>0$.

For $\lambda \$_{\lambda} 1=1$ it is necessary and sufficient that $\pi_{i i}+\pi_{12}=0$ or $\pi_{1}=\pi_{2}=\frac{1}{2}$, $\alpha=0$, respectively.

Proof. The two solutions $w_{i}$ in (10.6) may be chosen at will, but it is most convenient to let $w_{i}$ be the solution of (10.2) determined by the conditions

$$
w_{1}\left(\beta_{1}\right)=w_{2}\left(\beta_{2}\right)=1, \quad w_{1}\left(\beta_{2}\right)=w_{2}\left(\beta_{1}\right)=0 .
$$

(In other words, $w_{1}(x)=u_{* 2}(x) / u_{* 2}\left(\beta_{1}\right)$ and $w_{2}(x)=u_{* 1}(x) / u_{* 1}\left(\beta_{2}\right)$ where $u_{* i}$ is minimal at $\beta_{i}$.) We shall require the derivatives

$$
\begin{array}{ll}
\omega_{11}=-w_{1}^{+}\left(\beta_{1}\right), & \omega_{12}=w_{1}^{-}\left(\beta_{2}\right), \\
\omega_{21}=-w_{2}^{+}\left(\beta_{1}\right), & \omega_{22}=w_{2}^{-}\left(\beta_{2}\right) .
\end{array}
$$

It is clear that all these quantities depend on $\lambda$. 
Lemma 10.1. The matrix $\Omega$ of the elements $\omega_{i j}$ satisfies the conditions

$$
\omega_{11}>0, \quad \omega_{22}>0, \quad \omega_{12}=\omega_{21}<0 .
$$

$A s \lambda \rightarrow \infty$

$$
\omega_{i i} \rightarrow \infty, \quad \omega_{12} \rightarrow 0 .
$$

Proof. By (5.7) the Wronskian $w_{1} w_{2}^{\prime}-w_{1}^{\prime} w_{2}$ is constant. At the left boundary it reduces to $-\omega_{21}$, at the right to $-\omega_{12}$. Hence $\omega_{12}=\omega_{21}$. The signs in (10.14) are determined by the fact that $w_{1}$ decreases, while $w_{2}$ increases. Finally, if $\beta_{1}<x<x+h<\beta_{2}$, then (10.2) and the convexity of $w_{2}$ imply

$$
1 \geqq w_{2}(x+h) \geqq \lambda \int_{x+}^{x+h-} w(s) d m(s) \geqq \lambda w^{+}(x) \int_{x+}^{x+h-}(s-x) d m(s),
$$

and it follows that $w^{+}(x) \rightarrow 0$ as $\lambda \rightarrow \infty$ for each fixed $x<\beta_{2}$. This implies that $\omega_{12} \rightarrow 0$ and also that $\omega_{22} \rightarrow \infty$. The latter can be concluded equally from the fact that the Wronskian is constant.

Proof of Theorem 10.1(a). For each $f \in \mathrm{H}_{m}$ we have from (10.6)

$$
F=F^{\mathrm{min}}+\sum_{i, j} p_{i j}(\lambda) w_{i} \cdot\left(w_{j}, f\right),
$$

where $(g, f)$ is an abbreviation for the inner product

$$
(g, f)=\int_{\beta_{1}}^{\beta_{2}} g f d m .
$$

By Theorem 8.1 the function $F^{\text {min }}$ has the boundary values 0 , and hence

$$
F\left(\beta_{i}\right)=\sum_{j=1}^{2} p_{i j}(\lambda)\left(w_{j}, \lambda F-\mathfrak{U} F\right)
$$

Repeated integration by parts leads to

$$
\begin{array}{rl}
\lambda\left(w_{j}, F\right)=\int_{\beta_{1}}^{\beta_{2}} F d w_{j}^{\prime}=\omega_{j 1} F\left(\beta_{1}\right)+\omega_{j 2} & F\left(\beta_{2}\right) \\
& -(-1)^{j} F^{\prime}\left(\beta_{j}\right)+\left(w_{j}, \mathfrak{U} F\right),
\end{array}
$$

and (10.19) takes on the form

$$
F\left(\beta_{i}\right)=\sum_{j, k=1}^{2} p_{i j}(\lambda) \omega_{j k} F\left(\beta_{k}\right)-\sum_{j=1}^{2}(-1)^{j} p_{i j}(\lambda) F^{\prime}\left(\beta_{j}\right) .
$$

We have now to distinguish two cases.

Case I. The matrix $P(\lambda)$ with elements $p_{i j}(\lambda)$ is not singular. Premultiplying $(10.21)$ by $P^{-1}(\lambda)$, we are led to the boundary conditions (10.7) with the matrix $\Pi=\left(\pi_{i j}\right)$ given by

$$
\Pi=P^{-1}(\lambda)-\Omega .
$$

Thus each $F$ in the range satisfies (10.7). Conversely, suppose that $F \epsilon \mathbf{H}_{m}$, $\mathfrak{A} F \in \mathbf{H}_{m}$, and that (10.7) is true. Set $f=\lambda F-\mathfrak{U} F$ and $\bar{F}=\$_{\lambda} f$. Both 
$F$ and $\bar{F}$ satisfy (10.1), and hence $F-\bar{F}$ is a solution of (10.2), i.e. $F-\bar{F}=$ $\rho_{1} w_{1}+\rho_{2} w_{2}$. Now both $F$ and $\bar{F}$ satisfy (10.7), and hence $p_{i 1}(\lambda) \rho_{1}+$ $p_{i 2}(\lambda) \rho_{2}=0$. Since $P$ is nonsingular, this implies $\rho_{1}=\rho_{2}=0$, and it follows that (10.7) is a necessary and sufficient condition on the range.

Case II. The matrix $P(\lambda)$ is singular. Since it is symmetric, it is necessarily of the form

$$
P(\lambda)=T\left(\begin{array}{cc}
\pi_{1}^{2} & \pi_{1} \pi_{2} \\
\pi_{1} \pi_{2} & \pi_{2}^{2}
\end{array}\right) .
$$

Multiply the equations in (10.21) by $\pi_{2}$ and $-\pi_{1}$, respectively, to obtain

$$
\pi_{2} F\left(\beta_{1}\right)=\pi_{1} F\left(\beta_{2}\right) .
$$

If $\pi_{1}=\pi_{2}=0$ we have $F\left(\beta_{1}\right)=F\left(\beta_{2}\right)=0$. Otherwise each equation in (10.21) reduces to $(10.8)$ with

$$
T^{-1}=\alpha+\sum_{i, j=1}^{2} \omega_{i j} \pi_{i} \pi_{j} .
$$

This completes the proof of the first part.

Proof of Theorem 10.1(b). Prescribe an arbitrary symmetric matrix $\Pi=\left(\pi_{i j}\right)$. In view of (10.15) the diagonal elements in the matrix $\Pi+\Omega$ will be dominant for all $\lambda$ sufficiently large. It follows that for such $\lambda$ a matrix $P(\lambda)$ satisfying (10.22) exists, and the kernel (10.6) defines the transformation ${ }^{3}{ }_{\lambda}$ of the desired kind. This argument applies equally to (10.8) and (10.23).

Proof of Theorem 10.2(a). It is clear from (10.17) and (10.19) that ${ }^{3} \lambda$ is positive if, and only if, $p_{i j}(\lambda) \geqq 0$. Referring again to (10.15) we see that, for sufficiently large $\lambda$, the diagonal elements of $\Pi+\Omega$ are positive, while the corner elements approach $\pi_{12}$. For $P(\lambda)=(\Pi+\Omega)^{-1}$ to be positive, it is then necessary and sufficient that $\pi_{12} \leqq 0$. Similarly, in the case (10.23) the condition $\pi_{1} \pi_{2} \geqq 0$ is necessary and sufficient.

Proof of Theorem 10.2(b). We have

$$
\lambda \int_{\beta_{1}}^{\beta_{2}} w_{i} d m=\int_{\beta_{1}}^{\beta_{2}} d w_{i}^{\prime}=\omega_{i 1}+\omega_{i 2} .
$$

Substituting $f=1$ into (10.17) we find therefore

$$
\lambda F(x)=1-w_{1}(x)-w_{2}(x)+\sum_{i, j, k} w_{i}(x) p_{i j}(\lambda) \omega_{j k} .
$$

This shows that (10.9) holds if, and only if

$$
\sum_{j, k} p_{i j}(\lambda) \omega_{j k} \leqq 1, \quad i=1,2 .
$$

In other words, in order that $\oiint_{\lambda}$ be positive and (10.9) hold, it is necessary and sufficient that the row sums of $P(\lambda) \Omega$ be $\leqq 1$ and $P(\lambda) \geqq 0$. If (10.10) holds, then $P(\lambda) \geqq 0$ for all $\lambda \geqq 0$, and the row sums of $P(\lambda) \Pi$ are positive. However $P(\lambda) \Pi=1-P(\lambda) \Omega$, and therefore (10.10) is a sufficient condition 
for each $\lambda>0$. On the other hand, in the case of the boundary condition (10.7) the matrix $P(\lambda)$ converges to a diagonal matrix, and hence $P(\lambda) \Omega=$ $1-P(\lambda) \Pi$ can have row sums $\leqq 1$ only if $\Pi$ (which is independent of $\lambda$ ) has nonnegative row sums. Thus (10.10) is necessary and sufficient. The same argument applies, in a simpler form, to the case of the matrix (10.23). The two theorems are proved.

If $\beta_{2}=\infty$ the symbols $F\left(\beta_{2}\right)$ and $F^{-}\left(\beta_{2}\right)$ are senseless, and the same is true of formulas (10.12)-(10.13). However, the theorems and the proofs remain valid with a slight reinterpretation of the symbols. Instead of the basic Lemma 10.1 we use

LEMma 10.2. If $\beta_{2}=\infty$, let $w_{2}$ be that solution of (10.2) for which

$$
x^{-1} w_{2}(x) \rightarrow 1
$$

as $x \rightarrow \infty$,

and define $w_{1}$ similarly. Put

$$
\omega_{i 2}=\lim _{x \rightarrow \infty}\left\{x w_{i}^{\prime}(x)-w_{i}(x)\right\},
$$$$
i=1,2 \text {, }
$$

and define $\omega_{i 1}$ similarly in case $\beta_{1}=-\infty$ (and by (10.13) if $\left.\beta_{1}>-\infty\right)$. If both boundaries are active, these quantities are finite and satisfy (10.14)-(10.15).

Proof. By Theorem 6.3 the limit of $x^{-1} w(x)$ exists for each positive solution of (10.2). Now

$$
\lambda \int_{\beta}^{x} s w_{2} d m(s)=\int_{\beta}^{x} s d w_{2}^{\prime}(s)=\left.\left\{s w_{2}^{-}(s)-w_{2}(s)\right\}\right|_{\beta} ^{x} .
$$

From (6.9) it follows that $s w_{2}$ is square integrable over $\left(\beta_{1}, \beta_{2}\right)$ and therefore $x w_{2}(x)-w_{2}(x)$ approaches a limit monotonically as $x \rightarrow \infty$. Rewriting the Wronskian in the form

$$
\begin{aligned}
& w_{2}^{\prime}(x) w_{1}(x)-w_{1}^{\prime}(x) w_{2}(x) \\
& \quad=\left\{x w_{2}^{\prime}(x)-w_{2}(x)\right\} \cdot x^{-1} w_{1}(x)-\left\{x w_{1}^{\prime}(x)-w_{1}(x)\right\} \cdot x^{-1} w_{2}(x),
\end{aligned}
$$

we see the validity of the arguments in the proof of Lemma 10.1.

Consider now (10.17), and remember that the derivative of $F^{\text {min }}$ satisfies (7.14). It is seen at once that in the case of an active boundary at $\infty$ the limits

$$
\lim _{x \rightarrow \infty} x^{-1} F(x) \quad \text { and } \quad \lim _{x \rightarrow \infty}\left\{x F^{\prime}(x)-F(x)\right\}
$$

exist and are finite. With these quantities the proof of Theorem 10.1 applies without change and we have

Theorem 10.3. Theorem 10.1 applies also to the case $\beta_{2}=\infty$ provided the symbols $F\left(\beta_{2}\right)$ and $F^{\prime}\left(\beta_{2}\right)$ appearing in the boundary conditions are replaced by the limits appearing in (10.33). 
In the case of an operator $\mathfrak{A}_{\psi}$, Theorem 10.1 still applies with $F\left(\beta_{2}\right)$ and $F^{-}\left(\beta_{2}\right)$ to be replaced, respectively, by

$$
\lim _{x \rightarrow \beta_{2}} \frac{F(x)}{\phi(x)} \quad \text { and } \quad \lim _{x \rightarrow \beta_{2}} \phi^{2}(x) \cdot D_{x} \frac{F(x)}{\phi(x)},
$$

where $\phi$ is a solution of $\mathfrak{A}_{\psi} \phi=0$ which is at $\beta_{2}$ positive but not minimal.

The last formulation combines Theorems 10.1 and 10.2: in the case $\beta_{2}<\infty$ we set $\phi=1$; in the case $\beta_{2}=\infty$, however, $\phi=x$.

\section{Self-adjoint operators in closed intervals}

Consider a finite interval $I:\left(\beta_{1}, \beta_{2}\right)$ in which $m$ remains bounded: in other words, we suppose that both boundaries are finite and active. It is then natural to extend the measure $m$ to the closed interval $\bar{I}:\left[\beta_{1}, \beta_{2}\right]$ by attributing the weights $m_{1}$ and $m_{2}$ to the two points of the boundary. This leads to introducing the Hilbert space $\overline{\mathbf{H}}_{m}$ over $\bar{I}$ defined by the inner product

$$
(f, g)=\int_{\beta_{1-}}^{\beta_{2}+} f g d m=\int_{\beta_{1}+}^{\beta_{2}-} f g d m+\sum_{i=1}^{2} m_{i} f\left(\beta_{i}\right) g\left(\beta_{i}\right) .
$$

For sake of definiteness we shall suppose $m_{1}>0$ and $m_{2}>0$, although the argument applies equally when only one boundary point carries a positive weight.

So far the operator $\mathfrak{A}=D_{m} D_{x}$ is defined only in the interior of $\bar{I}$. We shall presently see that, essentially, there exists only one way of defining $\mathfrak{A}$ at the boundaries, provided we require that all functions in the domain of $\mathfrak{A}$ be continuous in $\bar{I}$.

A linear transformation $\overline{\mathcal{G}}_{\lambda}$ mapping each $f \epsilon \overline{\mathbf{H}}_{m}$ into a solution $F$ of $\lambda F-\mathfrak{A} F=f$ is necessarily of the form (10.5) where $\Phi^{(i)}$ is a functional in the enlarged Hilbert space $\overline{\mathbf{H}}_{m}$. If such $\bar{F}_{\lambda}$ is bounded and symmetric, it must be induced by a kernel $\bar{G}_{\lambda}$ obtained by extending the kernel $G_{\lambda}$ of (10.6) to the boundaries in accordance with the requirement that $F$ be continuous. Supposing that the solutions $w_{i}$ are specified by (10.12) we shall have

$$
\begin{array}{rlrl}
\bar{G}_{\lambda}(x, y) & =G_{\lambda}(x, y) & \beta_{1}<x, y<\beta_{2} \\
\bar{G}_{\lambda}\left(\beta_{i}, y\right) & =p_{i 1}(\lambda) w_{1}(y)+p_{i 2}(\lambda) w_{2}(y), & & \beta_{1}<y<\beta_{2} \\
\bar{G}_{\lambda}\left(x, \beta_{j}\right) & =p_{1 j}(\lambda) w_{1}(x)+p_{2 j}(\lambda) w_{2}(x), & & \beta_{1}<x<\beta_{2} \\
\bar{G}_{\lambda}\left(\beta_{i}, \beta_{j}\right) & =p_{i j}(\lambda), & & i, j=1,2 .
\end{array}
$$

(For example, the function $f$ defined by $f(x)=0$ for $x<\beta_{2}$ and $f\left(\beta_{2}\right)=1$ is mapped into $p_{11}(\lambda) w_{1}+p_{21}(\lambda) w_{2}$.)

The matrix $P(\lambda)$ of the coefficients $p_{i j}(\lambda)$ completely determines the transformation $\overline{G_{\lambda}}$. Given $\overline{F_{\lambda}}$, the operator $\mathfrak{A}$ at $\beta_{i}$ is defined for each $F$ in the range of $\bar{F}_{\lambda}$ by $\mathfrak{A} F\left(\beta_{i}\right)=\lambda F^{\prime}\left(\beta_{i}\right)-f\left(\beta_{i}\right)$. Formally this definition depends on $\lambda$. However, repeating the argument of Section 10 we are able to find all 
possible ranges, that is, all possible definitions of $\mathfrak{A}$. Given a possible range $R$ (i.e. a possible definition), we can construct the corresponding $\overline{\mathbb{S}}_{\lambda}$.

THeOREM 11.1. The Theorems 10.1 and 10.2 remain valid for the enlarged Hilbert space with inner product (11.1) provided that the boundary conditions (10.7) are replaced by

$$
\begin{aligned}
-F^{+}\left(\beta_{1}\right)+\pi_{11} F\left(\beta_{1}\right)+\pi_{12} F\left(\beta_{2}\right)+m_{1} \mathfrak{A} F\left(\beta_{1}\right) & =0 \\
F^{-}\left(\beta_{2}\right)+\pi_{21} F\left(\beta_{1}\right)+\pi_{22} F\left(\beta_{2}\right)+m_{2} \mathfrak{I} F\left(\beta_{2}\right) & =0,
\end{aligned}
$$

and (10.8) by

$$
\begin{aligned}
\alpha \pi_{1}^{-1} F\left(\beta_{1}\right)=\alpha \pi_{2}^{-1} F\left(\beta_{2}\right)=\pi_{1} F^{+}\left(\beta_{1}\right)- & \pi_{2} F^{-}\left(\beta_{2}\right) \\
& +\pi_{1} \mathfrak{2} F\left(\beta_{1}\right)+\pi_{2} \mathfrak{I} F\left(\beta_{2}\right) .
\end{aligned}
$$

It should be observed that when $m_{1} \neq 0, m_{2} \neq 0$, equations (11.3) represent a definition of $\mathfrak{A}$ at the boundary rather than "boundary conditions". The classical boundary conditions appear as a degenerate limiting case. The theorem asserts that, for sufficiently large $\lambda$, an operator $\mathfrak{S}_{\lambda}$ inverting $\lambda-\mathfrak{A}$ and acting from $\mathbf{H}_{m}$ to continuous functions is possible only if $\mathfrak{A}$ is defined as indicated. In the case $m_{2}=0$ the boundary point $\beta_{2}$ has measure zero, and no definition of $\mathfrak{A}$ is required. However, nonuniqueness persists, and the range $R$ must be specified by means of a lateral condition.

The proofs of Section 10 carry over to the present situation almost without change. In the construction of $\mathbb{S}_{\lambda}$ to given boundary conditions the relation (10.22) defining the matrix $P(\lambda)$ in the nonsingular case now takes on the form

$$
P^{-1}(\lambda)=\Pi+\Omega+M, \quad M=\left(\begin{array}{cc}
m_{1} & 0 \\
0 & m_{2}
\end{array}\right) .
$$

In the singular case of a matrix of the form (10.23) the coefficient $T$ is now given by

replacing (10.25).

$$
T^{-1}=\alpha+\sum_{i, j=1}^{2} \omega_{i j} \pi_{i} \pi_{j}+\lambda \sum_{i=1}^{2} m_{i} \pi_{i}^{2},
$$

\section{Positive operators in $\mathrm{C}$}

We turn to the problem of finding the general form of the solutions of $\lambda F-\mathfrak{A} F=f$ when $f, F$ are bounded continuous functions, $\mathfrak{A}=D_{m} D_{x}$, $\lambda>0$. Theorem 7.1 enables us to translate the results into statements concerning the general operator $\mathfrak{A}_{\psi}$.

The minimal Green function of Section 8 provides a solution $F^{\text {min }}$. This solution is unique except if at least one boundary is finite and either active or semiactive. In this case new solutions $F$ are formed by adding to $F^{\min }$ a bounded solution $w$ of $\lambda w-\mathfrak{A} w=0$. Such a $w$ has finite limits at both boundaries, and therefore $F$ and $\mathfrak{A} F$ have finite limits at $\beta_{i}$ whenever $f(x)$ 
tends to a limit as $x \rightarrow \beta_{i}$. Thus we are dealing with linear transformations from $\mathbf{C}$ to $\mathbf{C}$ (see (1.17)): the operator $\mathfrak{A}$ is defined at the boundary points by the requirement of continuity.

The object of our study is linear transformations $\omega_{\lambda}(\lambda>0)$ mapping each $f \epsilon \mathbf{C}$ into a solution $\mathfrak{S}_{\lambda} f=F$ of $\lambda F-\mathfrak{A} F=f$ such that

$$
0 \leqq f \leqq 1 \quad \text { implies } \quad 0 \leqq \lambda F \leqq 1 .
$$

Our problem is to construct the most general family $\left\{\mathcal{G}_{\lambda}\right\}$ of such transformations having a common range $R$. The restriction $\mathfrak{A} / R$ of $\mathfrak{A}$ to $R$ is then an operator independent of $\lambda$, and

$$
\mathfrak{S}_{\lambda}=(\lambda-\mathfrak{H} / R)^{-1}
$$

coincides with the resolvent of $\mathfrak{Q} / R$. In the language of semigroup theory, $\mathfrak{U} / R$ generates a transition semigroup, and we are constructing the most general transition semigroup. This has important applications in the theories of diffusion and Markov chains.

We proceed to describe the general form of the possible ranges $R$ and then to construct the corresponding $\mathbb{B}_{\lambda}$.

Definition 12.1. By boundary conditions of the general type we mean two independent $t^{19}$ equations

$$
\begin{aligned}
& \rho_{1} F\left(\beta_{1}\right)-\sigma_{1} F^{+}\left(\beta_{1}\right)+\tau_{1} \mathfrak{A} F\left(\beta_{1}\right)+\int_{\beta_{1}}^{\beta_{2}} F^{\prime}(x) \zeta_{1}(x) d x=0, \\
& \rho_{2} F\left(\beta_{2}\right)+\sigma_{2} F^{-}\left(\beta_{2}\right)+\tau_{2} \mathfrak{A} F\left(\beta_{2}\right)+\int_{\beta_{1}}^{\beta_{2}} F^{\prime}(x) \zeta_{2}(x) d x=0,
\end{aligned}
$$

where

$$
\begin{aligned}
\rho_{i} & \geqq 0, \quad \sigma_{i} \geqq 0, \quad \tau_{i} \geqq 0, \\
\zeta_{1} \downarrow, \quad \zeta_{2} \uparrow, \quad \zeta_{i} & \geqq 0, \quad \int_{\beta_{1}}^{\beta_{2}} \zeta_{i}(x) d x<\infty,
\end{aligned}
$$

and, of course, $\rho_{i}+\sigma_{i}+\tau_{i}+\zeta_{i} \neq 0$.

Boundary conditions of the special type are two equations

$$
\begin{gathered}
F\left(\beta_{1}\right)=F^{\prime}\left(\beta_{2}\right), \\
\rho F\left(\beta_{i}\right)-\sigma_{1} F^{+}\left(\beta_{1}\right)+\sigma_{2} F^{-}\left(\beta_{2}\right) \\
+\tau_{1} \mathfrak{A} F\left(\beta_{1}\right)+\tau_{2} \mathfrak{A} F\left(\beta_{2}\right)+\int_{\beta_{1}}^{\beta_{2}} F^{\prime}(x) \zeta(x) d x=0,
\end{gathered}
$$

where $\rho>0, \sigma_{i}>0, \tau_{i}>0$,

$$
\zeta \uparrow, \quad \int_{\beta_{1}}^{\beta_{2}}|\zeta(x)| d x<\infty,
$$

and $\rho+\sigma_{1}+\sigma_{2}+\tau_{1}+\tau_{2}+\zeta \neq 0$.

\footnotetext{
19 Since $\zeta_{1}$ decreases and $\zeta_{2}$ increases, the two equations (12.3) are necessarily independent unless $\sigma_{i}=\tau_{i}=0$ and $\zeta_{i}=$ const. In this case (12.6a) holds.
} 
$A$ range $R$ of the general [special] type consists of all $F \in \mathbf{C}$ such that $\mathfrak{A} F \in \mathbf{C}$ and $F$ satisfies boundary conditions of the general [special] type.

Theorem 12.1. Suppose that $\left|\beta_{i}\right|<\infty$ and that both boundaries are active.

(a) Let $\left\{\$_{\lambda}\right\}\left(\lambda>\lambda_{0}\right)$ be a family of operators from $\mathbf{C}$ to $\mathbf{C}$ with a common range $R$ such that (12.1)-(12.2) hold. Then $R$ is necessarily ${ }^{20}$ of the form described in Definition 12.1.

(b) Conversely, to each $R$ of Definition 12.1 and each $\lambda>0$, there corresponds $a$ (S) whose range is $R$ and such that (12.1)-(12.2) hold.

(c) In order that $\lambda\left(\$_{\lambda} \mathbf{1}=1\right.$, it is necessary and sufficient that $\rho_{1}=\rho_{2}=0$ (or $\rho=0$ in the special case (12.6)).

Proof. (a) Set-up. As in the proof of Theorem 10.1, let $w_{i}$ be the solutions of $\lambda w-\mathfrak{A} w=0$ satisfying the boundary conditions $w_{i}\left(\beta_{i}\right)=1, w_{1}\left(\beta_{2}\right)=$ $w_{2}\left(\beta_{1}\right)=0$. We are again concerned with transformations of the form (10.3) where $\mathcal{S}_{\lambda}^{\mathrm{min}}$ corresponds to the minimal Green function and $\Phi_{f}^{(i)}$ is a bounded linear functional. Each such functional is represented by a function of bounded variation (signed measure) in the closed interval $\bar{I}:\left[\beta_{1}, \beta_{2}\right]$. To simplify the writing we introduce the following notation for the inner product:

$$
(f, d \zeta)=\int_{\beta_{1}-}^{\beta_{2}+} f d \zeta
$$

where $\zeta$ is of bounded variation (we always suppose $\zeta$ continuous on the right). Note that the integral contains contributions from the two boundaries.

Putting

$$
F_{\lambda}=\$_{\lambda} f \quad F_{\lambda}^{\min }=\mathfrak{S}_{\lambda}^{\min } f,
$$

we can rewrite (10.3) in the form

$$
F_{\lambda}=F_{\lambda}^{\min }+w_{i} \cdot\left(f, d \Phi_{1}^{(\lambda)}\right)+w_{2} \cdot\left(f, d \Phi_{2}^{(\lambda)}\right)
$$

where (for fixed $\lambda, i$ ) the functions $\Phi_{i}^{(\lambda)}$ are of bounded variation. Since $F_{\lambda}^{\min }\left(\beta_{i}\right)=0$, we have

$$
F_{\lambda}\left(\beta_{i}\right)=\left(f, d \Phi_{i}^{(\lambda)}\right) .
$$

Thus $S_{\lambda}$ is a positive transformation if, and only if, the $\Phi_{i}^{(\lambda)}$ are nondecreasing. Furthermore

$$
\lambda\left(S_{\lambda}^{\min } \mathbf{1}=1-w_{1}-w_{2}\right.
$$

20 Theorem 12.1 generalizes the main result of [3] concerning operators of the special form $a D_{x}^{2}+b D_{x}$. Unfortunately the formulation in [3] contains an error: Due to a notational confusion it was erroneously claimed that the functions $\zeta_{i}$ are necessarily bounded whereas, in reality, only their integrals need be finite. The old proof is more complicated and is completely superseded by the present one. 
which shows that $\lambda\left(\$_{\lambda} 1 \leqq 1\right.$ if, and only if, the variation of $\lambda \Phi_{i}^{(\lambda)}$ is $\leqq 1$, i.e.

$$
\lambda\left\{\Phi_{i}^{(\lambda)}\left(\beta_{2}+\right)-\Phi_{i}^{(\lambda)}\left(\beta_{1}-\right)\right\} \leqq 1 .
$$

Each pair of nondecreasing $\Phi_{i}^{(\lambda)}$ satisfying this condition defines a positive transformation $\mathcal{B S}_{\lambda}$ such that $\lambda\left(S_{\lambda} \mathbf{1} \leqq 1\right.$, and all transformations of the desired kind are obtained in this way. A function $F \in \mathbf{C}$ is in the range of this S $_{\lambda}$ if, and only if, $\mathfrak{A} F \in \mathbf{C}$ and

$$
F\left(\beta_{i}\right)=\left(\lambda F-\mathfrak{Y} F, d \Phi_{i}^{(\lambda)}\right),
$$$$
i=1,2 .
$$

In fact, the necessity of this condition is obvious from (12.11). Conversely, define $f=\lambda F-\mathfrak{A} F$ and $F_{\lambda}=\$_{\lambda} f$. Then $F-F_{\lambda}=w$ is a solution of $\lambda w-\mathfrak{A} w=0$ vanishing at both boundaries, and hence $F=F_{\lambda}$.

(b) Construction of the ranges. Suppose that for each $\lambda>\lambda_{0}$ we are given two nondecreasing $\Phi_{i}^{(\lambda)}$ of variation $\leqq \lambda^{-1}$ such that (12.10) defines a transformation $\$_{\lambda}$ with range $R$ independent of $\lambda$. We want to show that $R$ is defined by boundary conditions as described in Definition 12.1.

Choose a fixed $F \in R$. For each $\lambda>\lambda_{0}$ equation (12.14) holds. Letting $\lambda \rightarrow \infty$ through an appropriate sequence, we may suppose that $\lambda \Phi_{2}^{(\lambda)} \rightarrow \zeta_{2}$ where $\zeta_{2}$ is nondecreasing and of variation $\leqq 1$. (The convergence is to be interpreted in the usual way as convergence at all points of continuity of $\zeta_{2}$, i.e. as "weak star" convergence.) Then $F$ satisfies the boundary condition $F\left(\beta_{2}\right)=\left(F, d \zeta_{2}\right)$ which is obviously a special case of (12.3) except if it reduces to the tautology $F\left(\beta_{2}\right)=F\left(\beta_{2}\right)$, i.e. except when $\lambda \Phi_{2}^{(\lambda)}(x) \rightarrow 0$ for $x<\beta_{2}$ and $\lambda \Phi_{2}^{(\lambda)}\left(\beta_{2}+\right) \rightarrow 1$. In this case the limiting function $\zeta_{2}$ attributes unit mass to $\beta_{2}$ and mass zero to the interval $\left[\beta_{1}, \beta_{2}\right]$.

Let this be true. Then

$$
\lambda\left(\mathfrak{A} F, d \Phi_{2}^{(\lambda)}\right) \rightarrow \mathfrak{A} F\left(\beta_{2}\right) .
$$

An integration by parts permits us to rewrite (12.14) in the form

$$
\begin{aligned}
a(\lambda) F\left(\beta_{2}\right)+\int_{\beta_{1}}^{\beta_{2}} F^{\prime}(x) \cdot \lambda \Phi_{2}^{(\lambda)}(x) d x & \\
& +\left(\mathfrak{A} F, d \Phi_{2}^{(\lambda)}\right)=0, \quad 0 \leqq a(\lambda) \leqq 1,
\end{aligned}
$$

(where we disposed of the arbitrary additive constant by setting $\left.\Phi_{2}^{(\lambda)}\left(\beta_{1}-\right)=0\right)$. Finally put

$$
A(\lambda)=\lambda \int_{\beta_{1}}^{\beta_{2}} \Phi_{2}^{(\lambda)}(x) d x
$$

Letting $\lambda \rightarrow \infty$ through an appropriate sequence we may suppose that

$$
A^{-1}(\lambda) \lambda \Phi_{2}^{(\lambda)} \rightarrow \zeta
$$

where $\zeta \geqq 0$ is an increasing function and

$$
\int_{\beta_{1}}^{\beta_{2}} \zeta(x) d x=1-\sigma \leqq 1 .
$$




\section{Clearly}

$$
A^{-1}(\lambda) \cdot \int_{\beta_{1}}^{\beta_{2}} F^{\prime}(x) \cdot \lambda \Phi_{2}^{(\lambda)}(x) d x \rightarrow \int_{\beta_{1}}^{\beta_{2}} F^{\prime}(x) \zeta(x) d x+\sigma F^{-}\left(\beta_{2}\right)
$$

Divide (12.16) by $\sigma(\lambda)=a(\lambda)+A(\lambda)+\lambda^{-1}$, and let $\lambda \rightarrow \infty$ in such a way that (12.18) holds. Taking, if necessary, a further subsequence $\lambda \rightarrow \infty$ such that

$$
a(\lambda) \sigma^{-1}(\lambda) \rightarrow \rho_{2}, \quad A(\lambda) \sigma^{-1}(\lambda) \rightarrow \delta, \quad \lambda^{-1} \sigma^{-1}(\lambda) \rightarrow \tau_{2},
$$

we see that the second equation in (12.3) holds with $\zeta_{2}=\delta \zeta$ and $\sigma_{2}=\delta \sigma$. Since $\rho_{2}+\tau_{2}+\delta=1$, we have $\rho_{2}+\tau_{2}+\sigma_{2}+\zeta_{2} \neq 0$, and we have thus verified the validity of the two equations (12.3).

However, we have to consider the possibility that these two equations are linearly dependent, i.e. that both reduce to the statement $F\left(\beta_{1}\right)=F\left(\beta_{2}\right)$. This can occur only if $\Phi_{1}^{(\lambda)}$ and $\Phi_{2}^{(\lambda)}$ are the same and (12.10) takes on the special form

$$
F_{\lambda}=F_{\lambda}^{\min }+\left(w_{1}+w_{2}\right) \cdot\left(f, d \Phi^{(\lambda)}\right) .
$$

The above argument can now be repeated with trivial changes. The first passage to the limit $\lambda \Phi^{(\lambda)} \rightarrow \zeta$ will reduce to a tautology whenever $\zeta$ is constant in the interior of $\left(\beta_{1}, \beta_{2}\right)$ but attributes unit mass to the set consisting of the two boundary points. In this case the right side in (12.15) is to be replaced by $p_{1} \mathfrak{U} F\left(\beta_{1}\right)+p_{2} \mathfrak{Y} F\left(\beta_{2}\right)$, where $p_{i} \geqq 0, p_{1}+p_{2}=1$. In the integration by parts leading to (12.16) we replace ${ }^{21}$ the norming $\Phi^{(\lambda)}\left(\beta_{1}-\right)=0$ by $\Phi^{(\lambda)}(\beta)=0$ where $\beta$ is an arbitrary fixed point, $\beta_{1}<\beta<\beta_{2}$. The limiting function $\zeta$ is capable of negative values and may be unbounded at both ends.

(c) Uniqueness. We have shown that our ranges satisfy the described boundary conditions, but it remains to show that each $F$ satisfying the boundary conditions is an element of the range (assuming, of course, that $\mathfrak{Y}(F \in \mathbf{C})$. This is equivalent to saying that no solution $w \neq 0$ of $\lambda w-\mathfrak{A} w=0$ satisfies the boundary conditions. A direct verification of this assertion is easy, but not necessary for our purposes. In fact, we shall prove that for each $\lambda>0$ there exists exactly one transformation $\left(\xi_{\lambda}\right.$ whose range satisfies the prescribed boundary conditions. On the other hand, $\left(S_{\lambda}\right.$ is determined only up to a multiple $\Phi_{f} \cdot w$ of a bounded solution $w$ of $\lambda w-\mathfrak{A} w=0$ which satisfies the boundary conditions. It follows that no such solution $\neq 0$ can exist.

Before proceeding to the existence proof, we digress to give

(d) An alternative form ${ }^{22}$ of the boundary conditions. The boundary con-

${ }^{21}$ Otherwise (12.18) would reduce to $A^{-1}(\lambda) \Phi^{(\lambda)}(x) \rightarrow p_{1}$ for each $x \epsilon\left(\beta_{1}, \beta_{2}\right)$, and, instead of deriving a new boundary condition, we would once more verify that $F\left(\beta_{1}\right)=$ $F\left(\beta_{2}\right)$.

${ }_{22}$ It is equally possible to proceed with a direct calculation using the original form of the boundary conditions and the quantities $\omega_{i j}$ of (10.13). The new form of the boundary conditions has the advantage of leading to the general form (12.44) when no positivity is required. 
ditions (12.3) are equivalent to

$$
\sum_{j=1}^{2} \alpha_{i j} F\left(\beta_{j}\right)+\tau_{\imath} \mathfrak{A} F\left(\beta_{i}\right)+\int_{\beta_{1}}^{\beta_{2}} \mathfrak{A} F(x) \cdot z_{i}(x) \cdot d m(x)=0
$$

where

$$
\left(\begin{array}{cc}
\alpha_{11} & \alpha_{12} \\
\alpha_{21} & \alpha_{22}
\end{array}\right)=\left(\begin{array}{cc}
\rho_{1}+c_{1} & -c_{1} \\
-c_{2} & \rho_{2}+c_{2}
\end{array}\right)
$$

and the $z_{i}$ are convex upward and such that

$$
\begin{aligned}
z_{i}\left(\beta_{i}\right)=\sigma_{i}, & z_{1}\left(\beta_{2}\right)=z_{2}\left(\beta_{1}\right)=0, \\
-z_{1}^{-}\left(\beta_{2}\right) \leqq c_{1}, & z_{2}^{+}\left(\beta_{1}\right) \leqq c_{2} .
\end{aligned}
$$

To pass from (12.3) to (12.23), define the constants $c_{i}$ by

$$
\int_{\beta_{1}}^{\beta_{2}}\left(c_{i}-\zeta_{i}\right) d x=\sigma_{i}
$$

and put

$$
z_{1}(x)=\int_{x}^{\beta_{2}}\left(c_{1}-\zeta_{1}(x)\right) d x, \quad z_{2}(x)=\int_{\beta_{1}}^{x}\left(c_{2}-\zeta_{2}(x)\right) d x .
$$

A simple integration by parts now transforms (12.3) into (12.23). Conversely, it is clear that the coefficients $\alpha_{i j}$ may be prescribed subject to the conditions $\alpha_{i 1}+\alpha_{i 2} \geqq 0, \alpha_{12} \leqq 0, \alpha_{21} \leqq 0$; and the convex $z_{i}$ may be chosen arbitrarily subject only to (12.25). Then an integration by parts leads backward from (12.23) to (12.3).

Similarly, the boundary condition (12.6b) may be rewritten in the form

$$
\rho F\left(\beta_{i}\right)+\tau_{1} \mathfrak{A} F\left(\beta_{1}\right)+\tau_{2} \mathfrak{A} F\left(\beta_{2}\right)+\int_{\beta_{1}}^{\beta_{2}} \mathfrak{A} F(x) \cdot z(x) \cdot d m(x)=0
$$

where

$$
z(x)=\sigma_{1}+\int_{\beta_{1}}^{x}(c-\zeta(x)) d x \quad z\left(\beta_{2}\right)=\sigma_{2} .
$$

(e) The construction. For a given $\lambda>0$ we propose to determine the $\Phi_{i}^{(\lambda)}$ in (12.10) so that the ensuing $\left(S_{\lambda}\right.$ will have a range satisfying the boundary conditions (12.23). For this purpose we introduce the notation

$$
Z_{i}(x)=\int_{\beta_{1}}^{\beta_{2}} G_{\lambda}^{\min }(x, y) z_{i}(y) d m(y)
$$

and

$$
q_{i j}=\lambda \int_{\beta_{1}}^{\beta_{2}} z_{i} w_{j} d m
$$

(remember that $m$ is defined in the open interval and hence that no contributions from the boundaries appear in (12.31)). 
Multiply (12.10) by $z_{i}$ and integrate to obtain the two equations

$$
\lambda \int_{\beta_{1}}^{\beta_{2}} F_{\lambda} z_{i} d m=\lambda \int_{\beta_{1}}^{\beta_{2}} f Z_{i} d m+\sum_{j=1}^{2} q_{i j} \cdot\left(f, d \Phi_{j}^{(\lambda)}\right) .
$$

By using the relation $\mathfrak{A} F_{\lambda}=\lambda F_{\lambda}-f$ it becomes evident that $F_{\lambda}$ satisfies the boundary conditions (12.23) if, and only if,

$$
\sum_{j=1}^{2} M_{i j}\left(f, d \Phi_{j}^{(\lambda)}\right)=\int_{\beta_{1}}^{\beta_{2}} f \cdot\left\{z_{i}-\lambda Z_{i}\right\} d m+\tau_{i} f\left(\beta_{i}\right)
$$

where the matrix $M$ is defined by

$$
\begin{aligned}
M=\left(\begin{array}{cc}
\alpha_{11}+q_{11}+\lambda \tau_{1} & \alpha_{12}+q_{12} \\
\alpha_{21}+q_{21} & \alpha_{22}+q_{22}+\lambda \tau_{2}
\end{array}\right) & \\
& =\left(\begin{array}{cc}
\rho_{1}+\lambda \tau_{1}+c_{1}+q_{11} & -c_{1}+q_{12} \\
-c_{2}+q_{21} & \rho_{2}+\lambda \tau_{2}+c_{2}+q_{22}
\end{array}\right) .
\end{aligned}
$$

We next prove that for each $\lambda>0$ this matrix possesses an inverse $M^{-1}$ with elements $M_{i j}^{-1} \geqq 0$.

Clearly $M_{i i}>0$. Remembering that $w_{1}\left(\beta_{2}\right)=0$ and $z_{2}\left(\beta_{1}\right)=0$ we get

$$
M_{21}=-c_{2}+\int_{\beta_{1}}^{\beta_{2}} z_{2} d w_{1}^{\prime}=-c_{2}-\int_{\beta_{1}}^{\beta_{2}} w_{1}^{\prime} \cdot z_{2}^{\prime} \cdot d x .
$$

Now $w_{1}^{\prime}<0$, while $z_{2}^{\prime}$ decreases from $z_{2}^{+}\left(\beta_{1}\right) \leqq c_{2}$. It follows that

$$
M_{21}<-c_{2}+c_{2} \int_{\beta_{1}}^{\beta_{2}}\left(-w_{1}^{\prime}\right) d x=0
$$

and similarly $M_{12}<0$. Hence $M_{i j}^{-1} \geqq 0$.

Premultiplication of (12.33) by $M^{-1}$ leads to an explicit form for $\Phi_{i}^{(\lambda)}$ and we have the following result:

In order that the range of the transformation (12.10) satisfy the boundary condition (12.3) [or the equivalent form (12.23)] it is necessary and sufficient that $\Phi_{i}^{(\lambda)}$ be absolutely continuous $(m)$ within the open interval $\left(\beta_{1}, \beta_{2}\right)$ with a density

$$
\frac{d \Phi_{i}^{(\lambda)}}{d m}=\sum_{j=1}^{2} M_{i j}^{-1}\left\{z_{j}-\lambda Z_{j}\right\}
$$

and that $\Phi_{i}^{(\lambda)}$ attaches weight $M_{i j}^{-1} \tau_{j}$ to the boundary $\beta_{j}$.

In the case of the special boundary condition (12.6) [or (12.28)] and the transformation (12.22), condition (12.37) is replaced by

$$
\frac{d \Phi^{(\lambda)}}{d m}=M^{-1}(z-\lambda Z)
$$

and the weights are $M^{-1} \tau_{i}$ where

$$
M=\rho+\lambda \tau_{1}+\lambda \tau_{2}+\lambda \int_{\beta_{1}}^{\beta_{2}}\left(w_{1}+w_{2}\right) z d m .
$$


Note. The above result may be formulated more elegantly in the case $\sigma_{i}>0$, by extending the measure $m$ to the boundary in such a way that (12.37) holds also on the boundary. We know that $Z_{i}$ vanishes at both boundary points. In view of (12.25) the function $z_{i}-\lambda Z_{i}$ equals $\sigma_{i}$ at $\beta_{i}$ and 0 at the other boundary. It follows that if $m$ is extended to the closed interval $\left[\beta_{1}, \beta_{2}\right]$ by attributing weight $m_{i}=\tau_{i} / \sigma_{i}$ to $\beta_{i}$, then $\Phi_{i}^{(\lambda)}$ is defined by the density (12.37) [or (12.38)] throughout the closed interval, and no special reference to $\beta_{i}$ is required.

(f) Verification of the norm conditions. Lemma 9.3 guarantees that $z_{j}-\lambda Z_{j} \geqq 0$. Since $M_{i j}^{-1} \geqq 0$, it follows that (12.37) defines nondecreasing $\Phi_{i}^{(\lambda)}$. Multiplying (12.12) by $z_{i}$ and integrating, we get, remembering (12.30) and (12.31),

$$
\lambda \int_{\beta_{1}}^{\beta_{2}}\left\{z_{i}-\lambda Z_{i}\right\} d m=q_{i 1}+q_{i 2} .
$$

For the special choice $f=1$ the right side in (12.33) reduces therefore to

$$
0 \leqq\left\{q_{i 1}+q_{i 2}+\tau_{i}\right\} \lambda^{-1}=\left\{M_{i 1}+M_{i 2}-\rho_{i}\right\} \lambda^{-1} \leqq\left\{M_{i 1}+M_{i 2}\right\} \lambda^{-1}
$$

with the equality sign holding only if $\rho_{i}=0$. Premultiplying the two equations $(12.41)$ by the positive matrix $M^{-1}$, we find that the variations of $\Phi_{i}^{(\lambda)}$ are $\leqq 1$, and equal to 1 only if $\rho_{1}=\rho_{2}=0$.

The same argument applies to (12.38) and completes the proof.

Theorem 12.2. In the case where $\beta_{2}$ is semiactive, Theorem 12.1 remains true except that in the boundary conditions $\sigma_{2}=0$.

Proof. The novel feature is that with a semiactive boundary $m\left(\beta_{2}\right)=\infty$, and therefore the boundedness of $\mathfrak{A} F$ no longer implies that $F^{\prime}$ is bounded. The above proof requires no change up to (12.19), but (12.20) makes no sense. Now

$$
F^{-}(x)-F^{+}(\beta)=\int_{\beta+}^{x-} \mathfrak{A} F \cdot d m,
$$

and hence

$$
F^{\prime}(x) m^{-1}(x) \rightarrow \mathfrak{A} F\left(\beta_{2}\right)
$$$$
x \rightarrow \beta_{2} .
$$

Thus the behavior of $F^{\prime}$ near $\beta_{2}$ is determined by $\mathfrak{A} F\left(\beta_{2}\right)$ instead of by $F^{-}\left(\beta_{2}\right)$. It is possible that for each $F$ in the range $\mathfrak{A} F\left(\beta_{2}\right)=0$. This then constitutes a special boundary condition of the form (12.3), and no further argument is required. Otherwise choose $F$ so that $\mathfrak{A} F\left(\beta_{2}\right)>0$. It is then obvious that (12.20) holds with $\sigma F^{-}\left(\beta_{2}\right)$ replaced by $\sigma^{\prime} \mathfrak{i} F\left(\beta_{2}\right)$. Thus the term containing $F^{-}\left(\beta_{2}\right)$ drops out. The actual construction of $\mathrm{B}_{\lambda}$ requires no change (and simplifies somewhat due to the absence of a term in the boundary condition).

Note. From (12.14) it is easy to deduce the general form of the boundary conditions when $\mathscr{S}_{\lambda}$ is not positivity preserving or norm-decreasing. Integrating twice by parts and adjusting the two arbitrary constants so as to 
eliminate the terms $F\left(\beta_{i}\right)$ we see easily that in general the range of $\mathcal{S}_{\lambda}$ will be determined by two equations of the form

$$
F\left(\beta_{i}\right)=\int_{\beta_{1}-}^{\beta_{2}+}(\mathfrak{A} F) \cdot d Z_{i} .
$$

The boundary conditions (12.22) are a special case and show the particular form required for $S_{\lambda}$ to be positive.

\section{The adjoint transformations}

Let $\mathbf{M}$ be the Banach space of functions of bounded variation (signed measures) on the closed interval $\bar{I}:\left[\beta_{1}, \beta_{2}\right]$, the total variation serving as norm. Considered as transformation from $\mathbf{C}$ to $\mathbf{C}$, each $B_{\lambda}$ has an adjoint (5) ${ }_{\lambda}^{*}$ mapping each $\pi \in \mathbf{M}$ into another element $\Pi=\mathfrak{S}_{\lambda}^{*} \pi \in \mathbf{M}$. By using the notation (12.8) for inner products, the adjoint $\mathcal{S}_{\lambda}^{*}$ is defined by $\left(\$_{\lambda} f, d \pi\right)=$ $(f, d \pi)$. If $B_{\lambda}$ is defined by a kernel

$$
\mathfrak{S}_{\lambda} f=\int_{\beta_{1}}^{\beta_{2}} G_{\lambda}(\cdot, y) f(y) d m(y)
$$

then the equation

$$
P(y)=\int_{\beta_{1}}^{\beta_{2}} G_{\lambda}(x, y) d \pi(x)
$$

defines a continuous $m$-density, and

$$
\Pi\left(x_{2}-\right)-\Pi\left(x_{1}+\right)=\int_{x_{1}}^{x_{2}} P(y) d m(y)
$$

defines the transform $\Pi=\left(S_{\lambda}^{*} \pi\right.$.

Consider first the case of a symmetric kernel $G_{\lambda}$, for example, the minimal Green function $G_{\lambda}^{\text {min }}$ of Section 8. If $\pi$ has a continuous density $p=d \pi / d m$, then $P=\$_{\lambda} p$, and hence

$$
\lambda P-\mathfrak{U} P=p .
$$

Integrating with respect to $m$ we find

$$
\lambda \Pi-D_{x} P=\pi+\text { const. }
$$

In other words, $\mathfrak{H}^{*}=D_{x} D_{m}$ appears as the formal adjoint of the differential operator $\mathfrak{A}=D_{m} D_{x}$. It is easily verified by integration by parts that (13.6) holds almost everywhere when $G$ is an arbitrary Green function and $\pi$ an arbitrary function of bounded variation.

The minimal Green function $G_{\lambda}^{\mathrm{min}}$ provides for each $\lambda>0$ a solution of (13.6) for given $\pi \in \mathbf{M}$. This solution is unique except if there exists a $v$ of bounded variation such that almost everywhere $(m)$

$$
\lambda v-D_{x} D_{m} v=0 \text {. }
$$


In this case it is readily seen that $u=D_{m} v$ is a solution of

$$
\lambda u-D_{m} D_{x} u=0, \quad \int_{\beta_{1}}^{\beta_{2}}|u| d m<\infty
$$

and, conversely, each $u$ satisfying (13.8) gives rise to a solution of (13.7) which is of bounded variation. Referring to Section 6 we see that the case of nonuniqueness arises if, and only if, a boundary $\beta_{i}$ is either active or infinite and semiactive.

Thus an active boundary causes nonuniqueness for the inversion both of $\lambda-\mathfrak{A}$ in $\mathbf{C}$ and for $\lambda-\mathfrak{H}^{*}$ in $\mathbf{M} ;$ a semiactive boundary $\beta_{i}$ causes nonuniqueness in the first case when $\left|\beta_{i}\right|<\infty$, and in the second case when $\left|\beta_{i}\right|=\infty$.

Whenever nonuniqueness occurs, the range $R^{*}$ of $\circlearrowleft_{\lambda}^{*}$ is determined by appropriate boundary conditions. With symmetric kernels these boundary conditions are the same in the spaces $\mathbf{C}$ and $\mathbf{M}$ so that no further discussion is required. However, an altogether new situation confronts us when $\mathfrak{S}_{\lambda}$ has an unsymmetric kernel $G$.

For sake of definiteness let us consider the transformation $B_{\lambda}$ of Theorem 12.1 determined by the boundary conditions (12.3). The formulas are simpler and more pleasing in the nondegenerate case $\sigma_{1} \neq 0, \sigma_{2} \neq 0$ when formal elegance can be achieved by extending the measure $m$ to the closed interval $\bar{I}:\left[\beta_{1}, \beta_{2}\right]$; by definition, the points $\beta_{i}$ carry the weights

$$
m_{1}=\tau_{1} / \sigma_{1}, \quad m_{2}=\tau_{2} / \sigma_{2},
$$

respectively. (The case $\sigma_{i}=0$ may be treated directly by the same method, or by a passage to the limit.)

Theorem 13.1. Let $m$ be defined in the closed interval $\bar{I}$ in accordance with (13.9). Let $\oiint_{\lambda}$ be that inverse to $\lambda-\mathfrak{A}, \lambda>0$, whose range $R$ is determined by the boundary conditions (12.3) supposing $0<m_{1}, m_{2}<\infty\left(\mathbb{S}_{\lambda}\right.$ is described in Theorem 12.1). Then:

(i) The adjoint $5_{\lambda}^{*}$ maps each function of bounded variation (signed measure) $\pi$ on $\bar{I}$ into $\Pi=\circlearrowleft_{\lambda}^{*} \pi$ such that $\Pi$ is absolutely continuous with respect to $m$ and its density $P=d \Pi / d m$ is continuous in $\bar{I}$. (It is given by (13.19)-(13.20).)

(ii) There exists an operator $\mathfrak{H}^{*}$, independent of $\lambda$, such that

$$
\left.\mathfrak{S}_{\lambda}^{*}=(\lambda-9)^{*}\right)^{-1}
$$

(i.e. $\mathfrak{S}_{\lambda}^{*}$ is the resolvent of $\mathfrak{A}^{*}$ and $\mathfrak{A}^{*}$ a formal adjoint to $\mathfrak{A}=D_{m} D_{x}$ ).

In the case when the monotone functions $\zeta_{1}$ and $\zeta_{2}$ occurring in the boundary conditions (12.3) as well as $\pi$ have densities

$$
\eta_{1}=-\frac{d \zeta_{1}}{d m}, \quad \eta_{2}=\frac{d \zeta_{2}}{d m}, \quad p=\frac{d \pi}{d m},
$$


the identity $\lambda \Pi-\mathfrak{I}^{*} \Pi=\pi$ may be expressed in terms of the density ${ }^{23} P=$ $d \Pi / d m$ as follows:

$$
\lambda P(x)-D_{m} D_{x} P(x)-P\left(\beta_{1}\right) \sigma_{1}^{-1} \eta_{1}(x)-P\left(\beta_{2}\right) \sigma_{2}^{-1} \eta_{2}(x)=p(x)
$$

for $\beta_{1}<x<\beta_{2}$, and

$$
\lambda P\left(\beta_{k}\right)-P\left(\beta_{k}\right) \rho_{k} \tau_{k}^{-1}-m_{k}^{-1} \Lambda_{k} P=p\left(\beta_{k}\right), \quad k=1,2
$$

where the operator $\Lambda_{i}$ is defined by $y^{24}$

$$
\Lambda_{k} P=(-1)^{k} \lim _{x \rightarrow \beta_{k}}\left\{P^{\prime}(x)-P\left(\beta_{1}\right) \sigma_{1}^{-1} \zeta_{1}(x)+P\left(\beta_{2}\right) \sigma_{2}^{-1} \zeta_{2}(x)\right\} .
$$

Note 1. Observe that in the interior $I$ the operator $\mathfrak{H}^{*}$ reduces to the differential operator $D_{x} D_{m}$ if, and only if, $\eta_{i}=0$, that is, if $\zeta_{i}=$ const. in the open interval $I$. In this case the boundary conditions (12.3) take on the form (11.3) known from the Hilbert space theory (except that the symmetry condition $\pi_{12}=\pi_{21}$ is no longer required). In other words, the classical boundary conditions with the added terms $\tau_{i} \mathfrak{A} F\left(\beta_{i}\right)$ are the only boundary conditions making the adjoint $\mathfrak{P}^{*}$ again a differential operator.

Note 2. Integrating (13.12) and adding to (13.13) we find the identity

$$
\lambda \int_{\beta_{1}-}^{\beta_{2}+} P(y) d m(y)+\sum_{k=1}^{2} P\left(\beta_{k}\right) \sigma_{k}^{-1} \rho_{k}=\int_{\beta_{1}-}^{\beta_{2}+} p(y) d m(y) .
$$

Since $\mathscr{S}_{\lambda}^{*}$ is positive, (13.15) shows that $\lambda\left\|\mathcal{S S}_{\lambda}^{*}\right\|=1$ if, and only if, $\rho_{1}=\rho_{2}=0$. This, of course, follows also from Theorem 12.1, (c).

Proof. The transformation ${\left(S_{\lambda}\right.}_{\lambda}$ is induced by the kernel

$$
G_{\lambda}(x, y)=G_{\lambda}^{\min }(x, y)+\sum_{i, j=1}^{2} w_{i}(x) M_{i j}^{-1}\left\{z_{j}(y)-\lambda Z_{j}(y)\right\}
$$

where $\beta_{1} \leqq x, y \leqq \beta_{2}$ and $G_{\lambda}^{\text {min }}$ is the minimal Green function for $A$ and the interval $I$ (see (12.37) and the note following (12.39)). In other words, the transform $\Pi=\mathfrak{S}_{\lambda}^{*} \pi$ has the density $P=d \Pi / d m$ defined by

$$
P(y)=P^{\min }(y)+\sum_{i, j=1}^{2}\left(w_{i}, d \pi\right) M_{i j}^{-1}\left\{z_{j}(y)-\lambda Z_{j}(y)\right\}
$$

where $\beta_{1} \leqq y \leqq \beta_{2}$ and

$$
P^{\min }(y)=\int_{\beta_{1}}^{\beta_{2}} G_{\lambda}^{\min }(x, y) d \pi(y)
$$

the notation $(f, d \pi)$ for inner products was introduced in (12.8).

${ }^{23}$ The explicit form of $\mathfrak{A}^{*}$ in the general case without densities is, of course, obtained by integrating (13.12) and introducing the original set functions $\Pi, \zeta_{i}, \pi$ instead of their densities. Observe that this integration changes the term $D_{m} D_{x} P$ into $D_{x} D_{m} \Pi$.

${ }^{24} \mathrm{~A}$ special case of Theorem 13.1 is treated in [3]. However, due to the error mentioned in footnote 20 only the case of bounded $\zeta_{i}$ was treated, and therefore the analogue to (13.13) in [3], Section 22, has a more restricted form. 
For $y=\beta_{j}$ we get from (13.17) (see again the note following (12.39))

$$
P\left(\beta_{j}\right)=\sum_{i=1}^{2}\left(w_{i}, d \pi\right) M_{i j}^{-1} \sigma_{j},
$$

and (13.17) may more conveniently be rewritten in the form

$$
P(y)=P^{\min }(y)+\sum_{j=1}^{2} P\left(\beta_{j}\right) \sigma_{j}^{-1}\left\{z_{j}(y)-\lambda Z_{j}(y)\right\} .
$$

The continuity of $P$ is obvious. To prove (13.12) we recall the definition (12.27) of $z_{j}$ according to which

$$
D_{x} z_{1}=-c_{1}+\zeta_{1}, \quad D_{x} z_{2}=c_{2}-\zeta_{2} .
$$

Thus $\eta_{j}=D_{m} D_{x} z_{j}$, by the definition (13.11). Since $\lambda P^{\min }-\mathfrak{A} P^{\min }=p$ and $\lambda Z_{j}-\mathfrak{Y} Z_{j}=z_{j}$, the assertion (13.12) follows on operating with $\mathfrak{A}$ on (13.20).

The relations (13.13) are less obvious. The minimal Green function is formed by means of the two solutions $w_{2}, w_{1}$, which are minimal at $\beta_{1}$ and $\beta_{2}$, respectively. They satisfy the boundary conditions $w_{1}\left(\beta_{2}\right)=w_{2}\left(\beta_{1}\right)=0$, $w_{i}\left(\beta_{i}\right)=1$, and their Wronskian equals $w_{2}^{+}\left(\beta_{1}\right)=-w_{1}^{-}\left(\beta_{2}\right)$. It follows (cf. (7.14)) that

$$
D_{x}^{+} P^{\min }(x)=-\frac{w_{1}^{+}(x)}{w_{1}^{-}\left(\beta_{2}\right)} \int_{\beta_{1}+}^{x+} w_{2}(y) d \pi(y)+\frac{w_{2}^{+}(x)}{w_{2}^{+}\left(\beta_{1}\right)} \int_{x+}^{\beta_{2}-} w_{1}(y) d \pi(y) .
$$

Hence

$$
(-1)^{j} \lim _{x \rightarrow \beta_{j}} D_{x} P^{\min }(x)=-\int_{\beta_{1}+}^{\beta_{2}-} w_{j}(y) d \pi(y) .
$$

Remembering that $\pi$ attaches weight $m_{i} p\left(\beta_{i}\right)$ to $\beta_{i}$ we can rewrite (13.23) as

$$
\begin{aligned}
(-1)^{j} \lim _{x \rightarrow \beta_{j}} D_{x} P^{\min }(x) & =m_{j} p\left(\beta_{j}\right)-\left(w_{j}, d \pi\right) \\
& =m_{j} p\left(\beta_{j}\right)-\sum_{k=1}^{2} P\left(\beta_{k}\right) \sigma_{k}^{-1} M_{k j}
\end{aligned}
$$

(cf. (13.19)).

Next, $Z_{i}=\mathfrak{S S}_{\lambda}^{\min } z_{i}$, and hence we have by analogy with (13.23)

$$
(-1)^{j} \lim _{x \rightarrow \beta_{j}} D_{x} Z_{i}(x)=-\int_{\beta_{1}+}^{\beta_{2}-} w_{j}(y) z_{i}(y) d m(y)=-\lambda^{-1} q_{i j}
$$

(using the notation (12.31)).

We now differentiate (13.20) with respect to $y$ using (13.24), (13.21), and (13.25). In this way we find for the operators defined in (13.14)

$$
\Lambda_{k} P=m_{k} p\left(\beta_{k}\right)+\sum_{i=1}^{2} P\left(\beta_{i}\right) \sigma_{i}^{-1}\left\{-M_{i k}+(-1)^{i+k} c_{k}+q_{i k}\right\} .
$$

Finally, by using the definition (12.34) of the matrix $M$ it is seen that within 
the braces we have the elements of a diagonal matrix, $\rho_{i}+\lambda \tau_{i}$, and thus (13.26) reduces to

$$
\Lambda_{k} P=m_{k} p\left(\beta_{k}\right)-\lambda P\left(\beta_{k}\right) m_{k}-P\left(\beta_{k}\right) \rho_{k} \sigma_{k}^{-1} .
$$

Dividing by $m_{k}$ we get (13.13).

To complete the proof of the theorem it remains to verify (13.10), namely, that (13.12)-(13.13) admit of only one solution. Now consider the $P\left(\beta_{j}\right)$ for the moment as two parameters. If these are given, (13.12) admits of exactly one solution such that $P(x) \rightarrow P\left(\beta_{j}\right)$ as $x \rightarrow \beta_{j}$. Equations (13.13) then represent two algebraic linear equations for the two unknowns $P\left(\beta_{1}\right)$ and $P\left(\beta_{2}\right)$. We know that to arbitrarily prescribed $p\left(\beta_{j}\right)$ there exists at least one solution, namely the one furnished by $\mathcal{S}_{\lambda}^{*}$. It follows that the determinant of the system can not vanish, and hence the solution is unique. The theorem is proved.

Note. We have shown that the operator $\mathfrak{A}^{*}$ is completely determined by (13.12)-(13.13) without any boundary conditions. In the limiting case $m_{2}=0$ the boundary $\beta_{2}$ carries no mass, and it is senseless to talk about a density $P=d \Pi / d m$ at the individual point $\beta_{2}$. However, $P\left(\beta_{2}\right)$ remains defined by the requirement of continuity $P\left(\beta_{2}\right)=\lim P(x)$ as $x \rightarrow \beta_{2}$. Multiply the second equation in (13.13) by $m_{k}$ and pass to the limit $m_{k} \rightarrow 0$. We get formally

$$
\rho_{2} P\left(\beta_{2}\right)+\sigma_{2} \Lambda_{2} P=0 \text {. }
$$

In this case it is senseless to talk about $\mathfrak{I}^{*}$ at $\beta_{2}$. However, the operator $\mathfrak{I}^{*}$ defined by (13.12)-(13.13) is now to be restricted by the boundary condition (13.28). Thus, exactly as in the case of the Hilbert space (see Section 11) the boundary conditions appear as a limiting case of the definition of operators on the boundary. Incidentally, when the functions $\zeta_{i}$ appearing in the boundary conditions (12.3) are bounded, then $\Lambda_{k} P$ reduces to a linear combination of $P^{\prime}\left(\beta_{k}\right), P\left(\beta_{1}\right), P\left(\beta_{2}\right)$, and (13.28) reduces to a boundary condition of the classical type.

\section{REFERENCES}

1. W. Feller, On second order differential operators, Ann. of Math. (2), vol. 61 (1955), pp. 90-105.

2. - On the intrinsic form for second order differential operators, to appear in Illinois J. Math., vol. 2 (1958).

3. - The parabolic differential equations and the associated semi-groups of transformations, Ann. of Math. (2), vol. 55 (1952), pp. 468-519.

4. W. Feller and H. P. McKean, Jr., A diffusion equivalent to a countable Markov chain, Proc. Nat. Acad. Sci. U. S. A., vol. 42 (1956), pp. 351-354.

5. H. P. McKean, JR., Elementary solutions for certain parabolic partial differential equations, Trans. Amer. Math. Soc., vol. 82 (1956), pp. 519-548.

6. M. H. Stone, Linear transformations in Hilbert space, Amer. Math. Soc. Colloquium Publications, vol. 15 (1932). 
7. H. WeyL, Über gewöhnliche Differentialgleichungen mit Singularitäten und die zugehörige Entwicklungen willkürlicher Funktionen, Math. Ann., vol. 68 (1910), pp. 220-269.

Added in proof. Cf. footnote 10.

8. A. D. Ventcelu, Semigroups of operators generated by a generalized differential operator of second order [in Russian], Doklady Akad. Nauk SSSR, vol. 111 (1956), pp. 269-272.

\section{Princeton University}

Princeton, New Jersey 